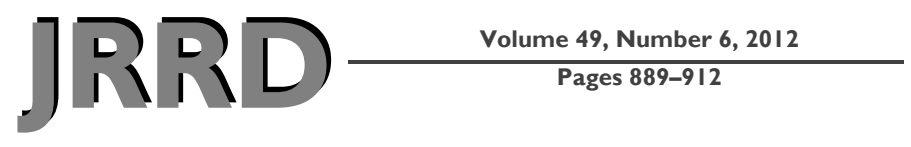

\title{
Center of mass acceleration feedback control of functional neuromuscular stimulation for standing in presence of internal postural perturbations
}

\author{
Raviraj Nataraj; ${ }^{*}$ Musa L. Audu, PhD; Ronald J. Triolo, PhD \\ Louis Stokes Department of Veterans Affairs Medical Center, Cleveland, $\mathrm{OH}$
}

\begin{abstract}
This study determined the feasibility and performance of center of mass (COM) acceleration feedback control of a neuroprosthesis utilizing functional neuromuscular stimulation (FNS) to restore standing balance to a single subject paralyzed by a motor and sensory complete, thoracic-level spinal cord injury. An artificial neural network (ANN) was created to map gain-modulated changes in total body COM acceleration estimated from body-mounted sensors to optimal changes in stimulation required to maintain standing. Feedback gains were systematically tuned to minimize the upper-limb (UL) loads applied by the subject to an instrumented support device during internally generated postural perturbations produced by volitional reaching and object manipulation. Total body COM acceleration was accurately estimated ( $>90 \%$ variance explained) from 2 three-dimensional (3-D) accelerometers mounted on the pelvis and torso. Compared with constant muscle stimulation employed clinically, COM acceleration feedback control of stimulation improved standing performance by reducing the UL loading required to resist internal postural disturbances by $27 \%$. This case study suggests that COM acceleration feedback could potentially be advantageous in a standing neuroprosthesis since it can be implemented with only a few feedback parameters and requires minimal instrumentation for comprehensive 3-D control of dynamic standing function.
\end{abstract}

Key words: acceleration, balance, center of mass, control system, feedback, functional neuromuscular stimulation, neuroprosthesis, posture, rehabilitation, spinal cord injury, standing.

\section{INTRODUCTION}

Neuroprostheses employing functional neuromuscular stimulation (FNS) have been clinically effective in restoring basic standing function following paralysis due to spinal cord injury (SCI) [1-3]. In most clinical systems, upright posture is maintained by constant supramaximal stimulation to the paralyzed musculature. Under constant stimulation, neuroprosthesis users are required to maintain balance by making postural corrections with their upper-limb (UL) loads on an assistive device or support surface such as a walker or countertop. Sustained UL loading can compromise the utility of standing with FNS by limiting the functional use of the hands and arms. To reduce user effort toward stabilization, feedback control of stimulation is necessary to produce the postural adjustments that facilitate augmented standing balance function. This study examined the performance of a new control system utilizing acceleration-based feedback to continuously adjust stimulation and reduce the UL loading required to maintain standing during internal postural perturbations generated during voluntary reaching activity.

Previous studies investigating feedback control of standing with FNS have focused on servo-type joint feedback at isolated joints. Measurable improvements in

\footnotetext{
Abbreviations: $3-\mathrm{D}=$ three-dimensional, $\mathrm{ANN}=$ artificial neural network, $\mathrm{AP}=$ anterior-posterior, $\mathrm{COM}=$ center of mass, FNS = functional neuromuscular stimulation, $\mathrm{I}=$ implant (stimulation), $\mathrm{ML}=$ medial-lateral, $\mathrm{S}=$ surface (stimulation), SCI = spinal cord injury, UL = upper-limb, SD = standard deviation, VAMC = Department of Veterans Affairs medical center. *Address all correspondence to Raviraj Nataraj; Louis Stokes VAMC, 10701 East Boulevard, Room C-15 (Motion Study Lab), Cleveland, OH 44106; 216-486-2867. Email: rxn25@case.edu http://dx.doi.org/10.1682/JRRD.2011.07.0127
} 
disturbance response at the knees [4-5], hips [6-7], or ankles [8] were reported. Furthermore, joint feedback facilitates full and explicit control of the system model from which solutions are autonomous and can focally adapt to changing system parameters (e.g., muscle fatigue) for neuroprosthetic standing [9]. However, joint feedback studies are typically conducted while joints not under feedback control were immobilized with mechanical bracing or constant stimulation. This is a significant clinical hurdle because functional standing requires movements to be generated by joint kinematics and multiarticulate muscle actions occurring in three-dimensional (3-D) space [10]. Consequently, current standing systems used clinically still do not employ feedback control. To advance toward clinical utility, a feedback control system for FNS standing balance should operate comprehensively, whereby synergistic muscle control is 3-D across the trunk, hip, knee, and ankle joints simultaneously and with minimal constraints.

We have previously investigated comprehensive control of standing with FNS in simulation utilizing feedback of joint kinematics [11] and linear acceleration of total body center of mass (COM) [12]. When compared with the clinical analog of constant maximal excitation of target muscle groups, both feedback control systems significantly reduced ( $>40 \%$ ) the UL loading required to resist postural perturbations. However, the joint feedback system required tuning a total of 18 distinct gain parameters composed of proportional and derivative feedback from nine individual joints compared with only 2 feedback parameters for COM acceleration in the anterior-posterior (AP) and medial-lateral (ML) dimensions. The amount of instrumentation for robust measurement and procedural tuning for that many joint feedback parameters may be too cumbersome and impractical for standard clinical usage.

COM acceleration potentially exhibits other features advantageous over joint feedback control for clinical usage of a standing neuroprosthesis, and acceleration has been previously shown as an effective means for assessing standing balance [13-15]. Firstly, its dynamic response makes it acutely sensitive to the inertial effects of rapidly acting perturbations and facilitates controller action before significant changes in standing posture occur. Secondly, COM acceleration provides a global representation of system dynamics, which has been implicated in standing balance control [16]. Finally, adequate measurement of COM acceleration for feedback control of standing may be plausible with only a few well-positioned sensors because per- turbed standing can be represented by simplified synergies [17-18] with higher concentration of body mass near the torso and pelvis [19].

In this single-subject case study, we present the development and performance evaluation of a control system utilizing COM acceleration feedback to modulate stimulation to the paralyzed musculature of a subject with complete paraplegia undergoing internal postural perturbations. Perturbations were classified as internal because they were volitionally generated by the subject herself through systematic reaching and manipulation of an object with one arm while stabilizing with the other arm. These perturbations are more representative of reaching tasks undergone during activities of daily living and are distinct from perturbations that may be applied externally [20]. The control system was tuned and evaluated according to the reduction in loading the participant applied with the stabilizing arm on a customized support device.

\section{METHODS}

A control system employing COM acceleration feedback was developed and evaluated for a single SCI subject with an implanted neuroprosthesis for standing function. The subject stabilized herself against disturbances to postural balance with her arms using a customized support device with instrumented handles. The control system (Figure 1) was composed of an artificial neural network (ANN) trained on COM acceleration feedback inputs and stimulation level outputs. These input-output data for training the ANN were created according to an optimization algorithm devised to determine the changes in stimulation levels necessary to produce targeted changes in COM acceleration from normal erect stance. During live controller operation, the ANN was driven by proportional gain-modulated feedback of the AP and ML components of COM acceleration. COM acceleration was estimated online by a linear regression model using inputs from body-mounted accelerometer measurements. Negative feedback gains were tuned to output stimulation patterns that produced effects on COM acceleration opposing those generated by postural perturbations while minimizing UL loading. Controller performance was evaluated according to the reduction in UL loading the user required to resist perturbations. 


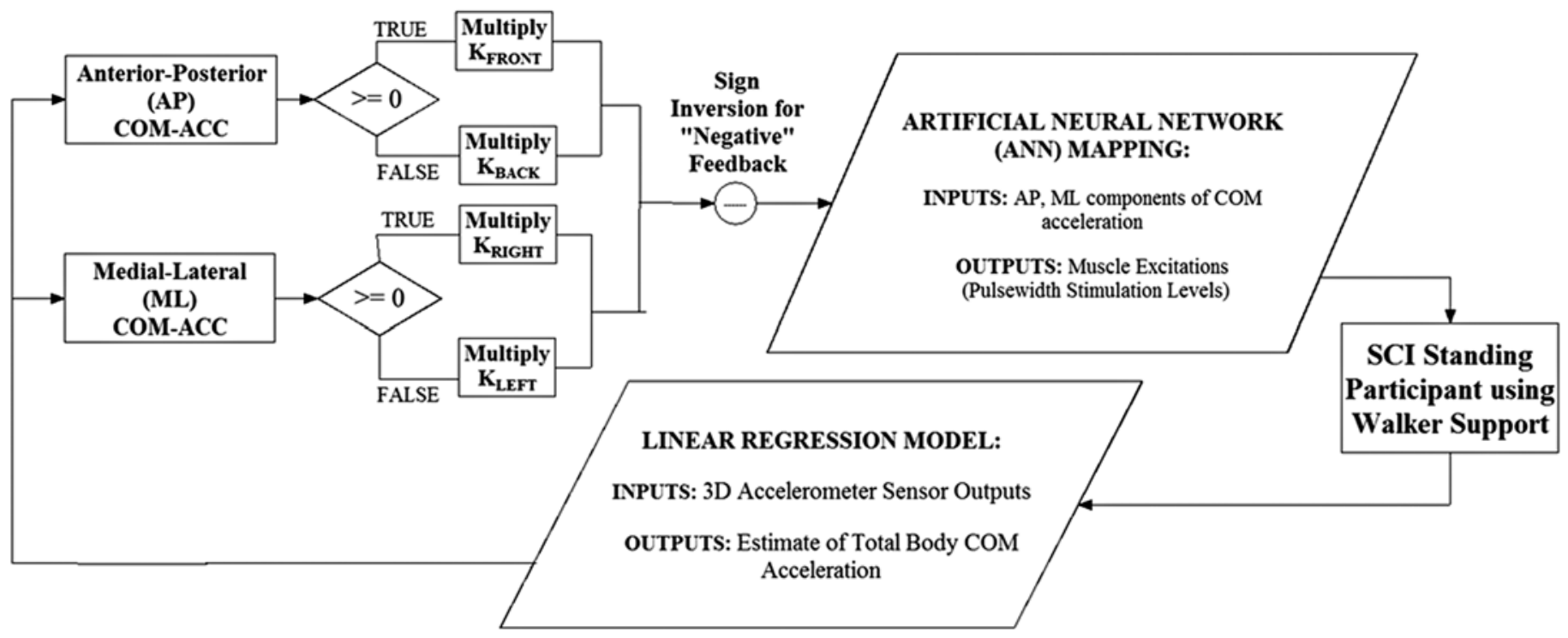

Figure 1.

Flow diagram for overall feedback control system. Proportional feedback of total body center of mass (COM) acceleration (ACC) drove acceleration-stimulation mapping represented by artificial neural network (ANN) to modulate muscle stimulation levels and assist individual with spinal cord injury $(\mathrm{SCl})$ to balance against postural disturbances while standing. $3 \mathrm{D}=$ three-dimensional.

Construction of the control system was composed of the following steps: (1) developing and validating a subject-specific acceleration-stimulation mapping represented by the ANN, (2) determining the parameters for a linear regression model that estimated COM acceleration used for controller feedback, (3) optimally tuning the feedback input gains, and (4) evaluating controller performance against internal perturbations.

\section{Subject and Control System Hardware}

The subject was a female who had thoracic-4 level complete paraplegia and was approximately $170 \mathrm{~cm}$ in height and $710 \mathrm{~N}$ (72.4 kg) in weight. The subject signed informed consent forms approved by the institutional review board of the Louis Stokes Cleveland Department of Veterans Affairs Medical Center (VAMC). She was actively using an FNS system for restoration of basic standing function at the time of all experimental data collections. The system included a surgically implanted stimulator-telemeter device and external control unit for modulating 16 independent channels of stimulation [21]. Stimulation was delivered via intramuscular electrodes to the following bilateral muscle groups (primary clinical anatomical function in italics): triceps surae (mainly gastrocnemius, ankle plantar flexion), tibialis anterior (ankle dorsiflexion), quadriceps (primarily vasti for knee extension), semimembranosus (i.e., hamstrings, hip extension), posterior adductor magnus (hip extension), gluteus maximus (hip extension), gluteus medius (hip abduction), and erector spinae (trunk extension). In order to introduce hip flexion and augment adduction and trunk extension, a custom external control unit that included an additional six channels of surface stimulation was utilized for control experiments. Surface stimulation was applied bilaterally to activate the erector spinae (electrode placed superior to the implanted electrode), rectus femoris (hip flexion, electrode placed at upper lateral thigh), and hip adductor (hip adduction, electrode placed at upper medial thigh) muscle groups. The ground electrode for surface stimulation was placed on the abdomen (side away from the implant) or bony landmarks at either the kneecap or anterior superior iliac spine. Exact locations of surface electrode placement varied session to session based on clinical observation according to which locations elicited the strongest stimulated responses while producing action largely along the desired anatomical planes of movement (i.e., hip flexion and trunk extension in sagittal plane, hip adduction in coronal plane).

Real-time control of stimulation was performed using software developed to run in MATLAB $^{\circledR} /$ Simulink $^{\circledR}$ 
(R14.3, The MathWorks, Inc; Natick, Massachusetts) in conjunction with the xPC Target toolbox (The MathWorks, Inc). The host computer responsible for building customized applications was a Windows ${ }^{\circledR}$ (Microsoft Corp.; Redmond, Washington) machine. The target computer that subsequently ran the applications in real time contained a Pentium Dual-Core $3 \mathrm{GHz}$ microprocessor (Intel Corp.; Santa Clara, California) with 2 GB of RAM (randomaccess memory). The host and target computers communicated via TCP/IP (transmission-control protocol/Internet protocol). Data were acquired using an NI PCI-6071E board (National Instruments Corp.; Austin, Texas). Stimulation consisted of charge balanced, biphasic constant current pulse trains. Current pulse amplitude, duration (i.e., pulse width), and frequency were controllable independently on each channel. All real-time controller parameter updates and stimulation frequencies were fixed at $20 \mathrm{~Hz}$. The pulse amplitude for implanted stimulation channels to the quadriceps and erector spinae was $2 \mathrm{~mA}$, which was sufficient to produce the knee and trunk extension required to stand erect without discomfort. The pulse amplitude for the remaining implanted stimulation channels was $20 \mathrm{~mA}$, the maximum allowable setting for this implant. Pulse amplitude for all surface stimulation channels was set at the maximum $100 \mathrm{~mA}$. Stimulation pulse width (0 to $250 \mu \mathrm{s})$ was adjusted to modulate muscle excitation levels and produce controller-mediated corrections.

To ensure the subject's ability to safely maintain near erect standing at all times, we determined minimum levels of stimulation pulse width for stimulation to the quadriceps (right $=140 \mu \mathrm{s}$, left $=150 \mu \mathrm{s}$ ), hamstrings (right $=119 \mu \mathrm{s}$, left $=167 \mu \mathrm{s}$ ), gluteus maximus (right $=32 \mu \mathrm{s}$, left $=$ $44 \mu \mathrm{s}$ ), and erector spinae (right $=85 \mu \mathrm{s}$, left $=113 \mu \mathrm{s}$ ). The minimal level for all other stimulation channels was $0 \mu \mathrm{s}$. These minimal levels were determined according to clinical observation of the subject's ability to comfortably maintain neutral erect standing with only one-arm support. Controller modulation of stimulation was never allowed to fall below these minimal levels to ensure the subject's ability to maintain standing position at all times during experimental sessions. This subject was able to maintain erect standing for 1-2 h each session. Stimulated muscles, most notably the knee extensors, had developed high fatigue resistance from diligent daily use of the implanted FNS system and a commercially available bicycle ergometer (ERGYS ${ }^{\circledR}$, Therapeutic Alliances Inc; Fairborn, Ohio) that combines surface stimulation with guided cyclic leg motion.

\section{Perturbation Testing}

Perturbation testing was conducted while the subject performed neutral bipedal standing (Figure 2) with each foot on a force platform (OR6-6-1000, Advanced Mechanical Technology, Inc; Watertown, Massachusetts). During upright standing, the participant balanced herself by applying corrective loads with her upper limbs on a customized support device. The device was composed of aluminum framing $\left(80 / 20^{\circledR}\right.$, Inc; Columbia City, Indiana) with adjustable left- and right-side block mounts that interfaced instrumented walker handles employing 6-degree of freedom load cells (MCW-500, Advanced Mechanical Technology, Inc). All collected analog data were sampled at $100 \mathrm{~Hz}$.

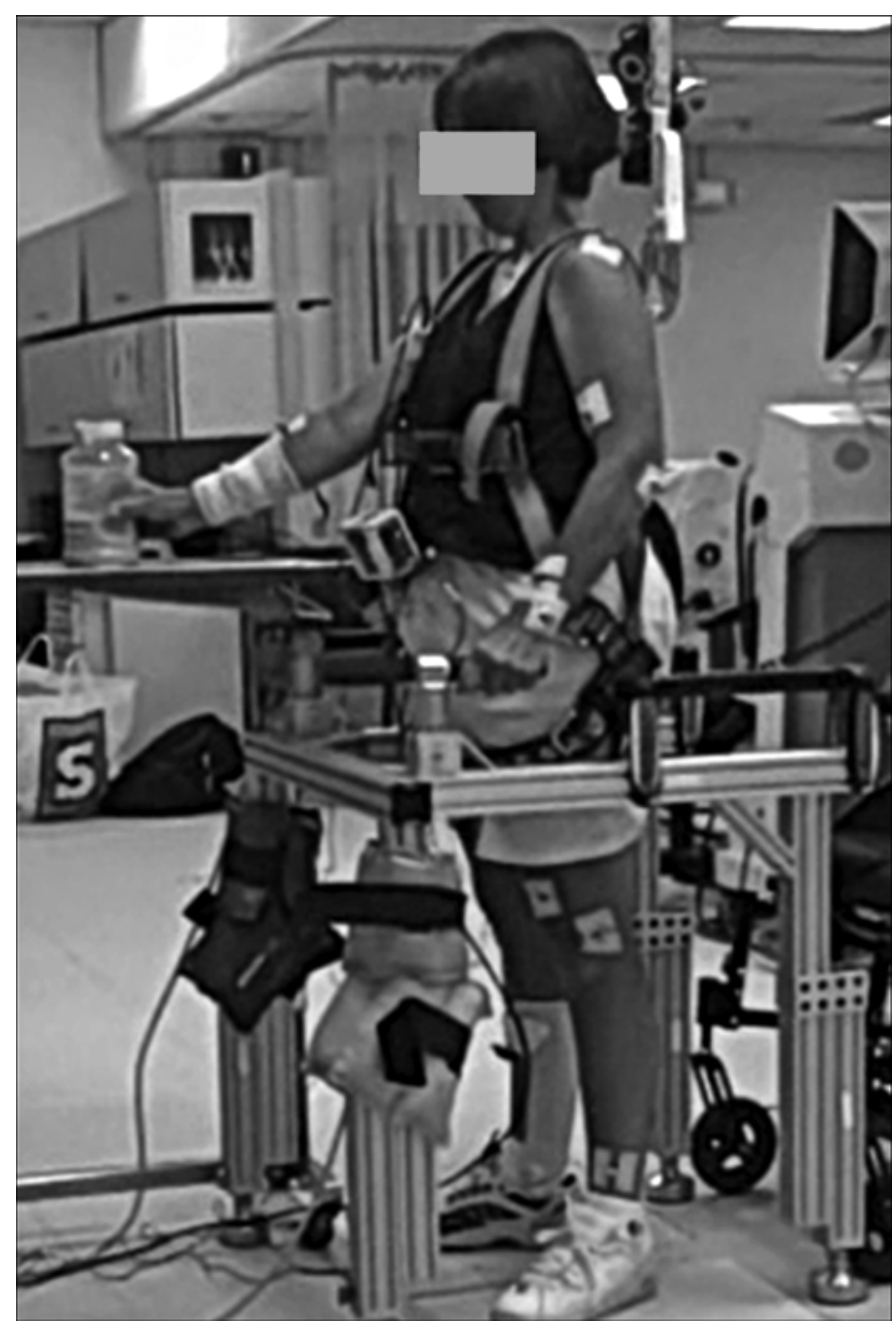

Figure 2.

Subject with spinal cord injury undergoing internal perturbations by volitionally moving object over level surface with one arm while stabilizing with other arm. 
Safety considerations included having the subject wear a standard fall prevention harness (distributed by McMasterCarr, Inc; Elmhurst, Illinois) connected to a structurally reinforced overhead hook via a safety lanyard (Guardian Fall Protection, Inc; Kent, Washington) meeting specifications set by the Occupational Safety and Health Administration.

Internal (i.e., volitionally generated) perturbations were produced with the subject standing and stabilizing herself with one arm on the instrumented support device while using the other arm to systematically manipulate an object horizontally across a smooth, level surface (Figure 2). The subject was left-side dominant and always used the left arm for support and the right arm for object manipulation. Each trial consisted of moving the object between two marked positions. The mode of movement was either sliding the object across the surface or picking it up and setting it down from one position to another. The surface was a heightadjustable, caster-wheel table placed directly in front of the subject just over the front of the support device. Table height was aligned just above the subject's waist, and the starting position of the object for all trials was 10 in. away from the subject directly in front of the right arm.

During internal perturbation trials, the object was moved in either the globally fixed AP or ML anatomical directions relative to the subject. Upon instruction, the subject moved the object to a target either $12 \mathrm{in.} \mathrm{left} \mathrm{along} \mathrm{the}$ ML axis (Figure 3(a)) or 6 in. forward along the AP axis (Figure 3(b)) from the starting position. From the new location (i.e., target away position), the subject subsequently returned the object to the starting position by using the same sliding or lifting motion. The subject was instructed to move the object briskly without overexertion. The object was either a 3-D accelerometer (CXL04LP3, Crossbow Technology; Milpitas, California) or a gripembedded plastic jar filled with $1 \mathrm{~kg}$ of sand. The subject held the accelerometer from above with the palm face down and slid it across the table between thin metal ruler guide rails secured to the table top. Starting and target positions were clearly marked on the guides to ensure consistent movements. The weighted plastic jar was instrumented with an accelerometer such that its primary measurement axis was aligned parallel to either the AP or ML axis of movement at the starting position. Movement of the jar was not constrained in any way, but the same ruled guide rails served as a visual reference for the subject to move the jar appropriately along the specified dimensions of movement. The plastic jar was oriented such that the subject could hold the grip with the right palm facing inward (i.e., to subject's left). The jar was either slid over the rulers or picked up and subsequently replaced between the starting and target positions on top of the rulers. The subject was instructed to maintain jar orientation as constantly as possible to ensure that the accelerations recorded were relative to the presumed globally fixed anatomical reference frame.

The subject systematically moved either the accelerometer alone or the instrumented jar according to a fixed auditory cue. The cue consisted of an easily audible metronome-paced beep that occurred at a frequency of 30 beats per minute (i.e., $0.5 \mathrm{~Hz}$ or 1 beep every $2 \mathrm{~s}$ ). The subject slid the accelerometer along the prescribed path on the counter from the starting position to the target position (i.e., 6 in. forward or 12 in. left) and immediately back to the starting position with each beep. This induced strong COM accelerations in both the front and back directions for movement along the AP dimension and both the right and left directions along the ML dimension for each single movement. However, when the subject was moving the $1 \mathrm{~kg}$ jar, this back and forth motion quickly produced fatigue. Therefore, the subject moved the weighted jar from its original location to its target location upon a single beep, but would only return the jar to its original location after the subsequent beep.

\section{Calculation of Center of Mass Acceleration for Feedback Control}

\section{Data Collection and Processing}

The subject underwent two pilot sessions of internal perturbation trials on separate days that included movement shifts of sliding the accelerometer, sliding the jar, and picking up and placing down the jar in both the AP and ML dimensions. Each movement shift in each dimension was repeated more than 100 times, resulting in more than 1,500 s of collected data containing more than 600 manual shifts. The 3-D positions of body segments were tracked according to a globally fixed reference frame with a Vicon ${ }^{\circledR}$ motion capture system (Vicon Motion Systems and Peak Performance, Inc; Oxford, United Kingdom). This motion capture system can collect data with respect to a globally fixed reference frame that can then be transformed to be aligned with presumed anatomical reference directions (e.g., AP, ML) for the subjects. This was important for reliable specification of the acceleration data used to subsequently develop the feedback control system. 


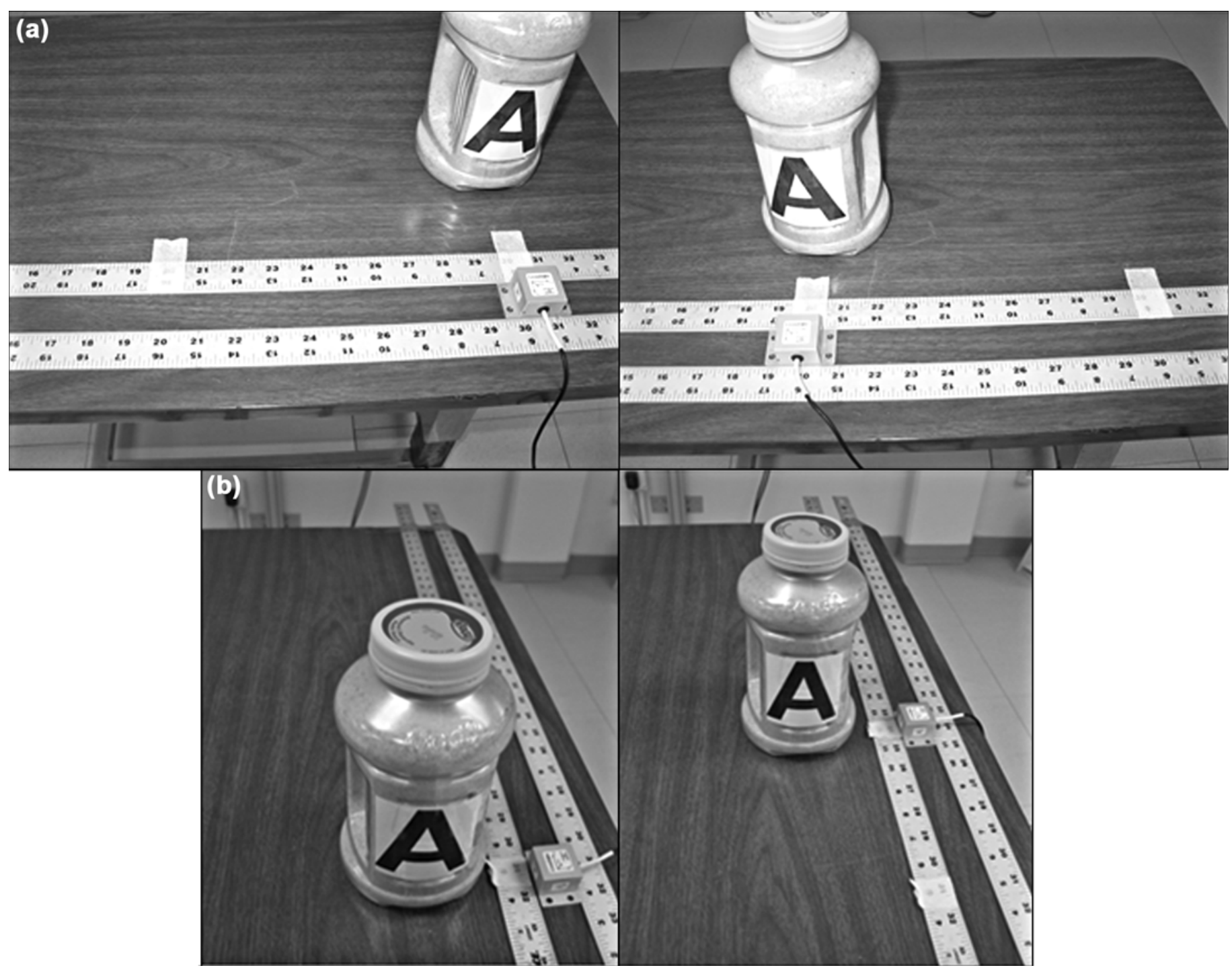

Figure 3.

Object set-up for internal perturbations in (a) medial-lateral and (b) anterior-posterior dimensions. Accelerometer and jar objects are aligned with starting position (left) and target away position (right).

All collected motion capture data were sampled at $100 \mathrm{~Hz}$. Retroreflective markers were placed on anatomical landmarks according to guidelines defined in the Vicon Plug-In Gait marker set (cervical 7 vertebra, clavicle, sacrum and bilateral shoulder, upper arm, elbow, forearm, wrist, anterior superior iliac spine, thigh, knee, tibia, ankle, heel, and toe). Marker positions were double differentiated [22] off-line to estimate segment COM accelerations according to segmental mass and COM location definitions from Winter [19] for the pelvis, trunk (assumed to include head), right and left upper arms, right and left forearms (include hands), right and left thighs, right and left shanks (i.e., lower legs), and right and left feet. The segmental COM accelerations were then used to calculate total body COM acceleration. The weighting of total body COM acceleration toward the torso and pelvis segments is consistent with theoretical representations of the standing system as either an inverted linkage in the AP plane or a four-bar linkage in series with the trunk segment in the ML plane [23].

\section{Determination of Linear Regression Models}

For online feedback control, outputs from bodymounted accelerometers were employed because real-time motion capture of Vicon position data was not employed 
and eventual clinical deployment would be facilitated by a minimal sensor approach. In turn, linear regression models were developed to utilize the accelerometer signals as inputs to accurately estimate the COM acceleration values that derive from Vicon motion capture, presumed to be the gold standard. From pilot data collected during internal perturbation sessions, a stepwise linear regression model (MATLAB Statistics Toolbox, The MathWorks, Inc) was determined that could accurately ( $>90 \%$ variance accounted for) estimate the AP and ML components of total body COM acceleration from the 3-D accelerations of discrete points on the body. For online feedback control, these inputs would be provided by 3-D accelerometers placed on these points.

For this study, two linear regression models were developed. The first used only the 3-D acceleration inputs from the two optimal locations determined for estimation during external postural perturbations as described in Nataraj [20]. The optimal placements for these accelerometers were the anterior side of the pelvis equidistant between the anterior superior iliac spines and the posterior torso between the sacrum and right shoulder at a point $\sim 40$ percent closer to the right shoulder. The placement offset from midline for the torso accelerometer was a byproduct of the asymmetric disturbance response for this particular subject. Another linear regression model additionally included as inputs the 3-D acceleration of a marker position placed at the wrist near where the object being moved would be located. Since the total body COM undergoes small changes in position during standing with high mass concentration toward the pelvis and torso, it was assumed that an additional acceleration input from the moving arm may be valuable in estimating total body COM acceleration during internal perturbations. The total body COM acceleration in the AP and ML dimensions served as the regression model outputs for both cases.

\section{Constructing Optimal Acceleration-Stimulation Control Map}

Construction of the acceleration-stimulation map for feedback control followed those steps outlined for the simulation control system summarized in Nataraj et al. [12]. Ultimately, an ANN was trained to output optimal changes in the levels of the stimulation (up to 16 implant channels plus 6 surface channels) according to 2 (AP, ML) COM acceleration feedback inputs.
Step 1

The first step was to determine the maximum COM acceleration $\left(a_{\mathrm{COM}}\right)$ that could be induced from quiet erect standing as a result of the maximum change in activation of individual muscle groups targeted by stimulation. In Nataraj et al. [12], model system equations of motion were used to determine maximal COM acceleration values induced as a result of maximal changes in muscle activation for each muscle group assuming the initial position and velocity states of the model correspond to a set point position for quiet upright standing. However, for live subject experimentation, because of the presence of muscle excitation-activation coupling delay [24] and insufficient quantitative description of our specific subject as a mechanical system, the changes in COM acceleration could not be explicitly determined from mathematical equations. Instead, surrogate values for $a_{\mathrm{COM}}$ were produced from live observed measurements.

The subject initially stood using the minimum stimulation levels previously described. These minimum baseline stimulation levels ( $M_{\text {base }}$ ) produced the minimum muscle activation levels necessary to generate basic support for upright standing according to clinical observation. From quiet erect standing, an instantaneous maximum change in stimulation level $\left(M_{\mathrm{max}}\right)$, the difference between the maximum and minimum levels, was applied to a single stimulation channel by using a pulse train duration of $750 \mathrm{~ms}$. The maximum stimulation level $\left(M_{\max }\right)$ for each corresponding muscle group equaled the maximum deliverable pulse width of $250 \mu$ s except for the right gastrocnemius and left tibialis anterior, whose maximum levels were set at 50 and $125 \mu$ s, respectively. Instantaneous changes in stimulation greater than these maximum levels produced postural changes that the subject found intolerable. Observing and respecting these thresholds of tolerance was important in order to manage the onset of both physical and mental fatigue. This ensured that the subject could perform with high vigilance throughout a 2-hour experimental session.

The maximum change (positive or negative) in COM acceleration in both the AP and ML dimensions occurring within a 500 ms window following onset of the stimulation pulse train was identified and recorded as the trial value of $a_{\mathrm{COM}}$ for each stimulated muscle group in each dimension. This was repeated for a total of 80 trials for each muscle group over 20 experimental sessions, each session occurring on a separate day. At least $4 \mathrm{~s}$ elapsed between individual trials and was found to be ample time for the subject 
to recover and return to quiet erect standing before onset of a subsequent trial. The average value across all 80 trials was assigned as the final $a_{\mathrm{COM}}$ for the given muscle group. An example trial is shown in Figure 4.

The 750 ms pulse train was predetermined as being the longest the subject could tolerate from quiet standing for all muscle groups. The $500 \mathrm{~ms}$ window was sufficiently long to consistently capture the initial maximum acceleration occurring because of change in stimulation prior to subsequent maximal accelerations resulting from the subject's reactions to stabilize with the arms. Pilot data showed the initial peak acceleration from quiet standing occurred on average $308 \pm 40 \mathrm{~ms}$ following onset of the stimulation pulse train. The same data showed that the maximum UL loading the participant applied to stabilize occurred on average $690 \pm 149 \mathrm{~ms}$ following onset of the stimulation pulse train.
Step 2

The maximal changes in muscle stimulation, $M_{\max }$, of individual muscle groups and the corresponding maximal changes in total body COM acceleration, $a_{\mathrm{COM}}$, were used to determine the optimal mapping to be driven for feedback control. An optimization algorithm was formulated to determine the optimal muscle stimulation levels to produce a given COM acceleration target. These targets assumed operation about the set point stance observed for quiet erect standing. For the optimization, the maximum stimulation level, $M_{\max }$, was normalized to 1 and the minimum muscle stimulation level used for maintenance of steady state standing, $M_{\text {base }}$, was normalized to 0 for each muscle group. Thus, any changes in muscle stimulation were bounded over $[0,1]$ for each muscle group and $M_{\max }$ simply equaled 1 . Only positive changes in stimulation were explored because the minimum levels were necessary

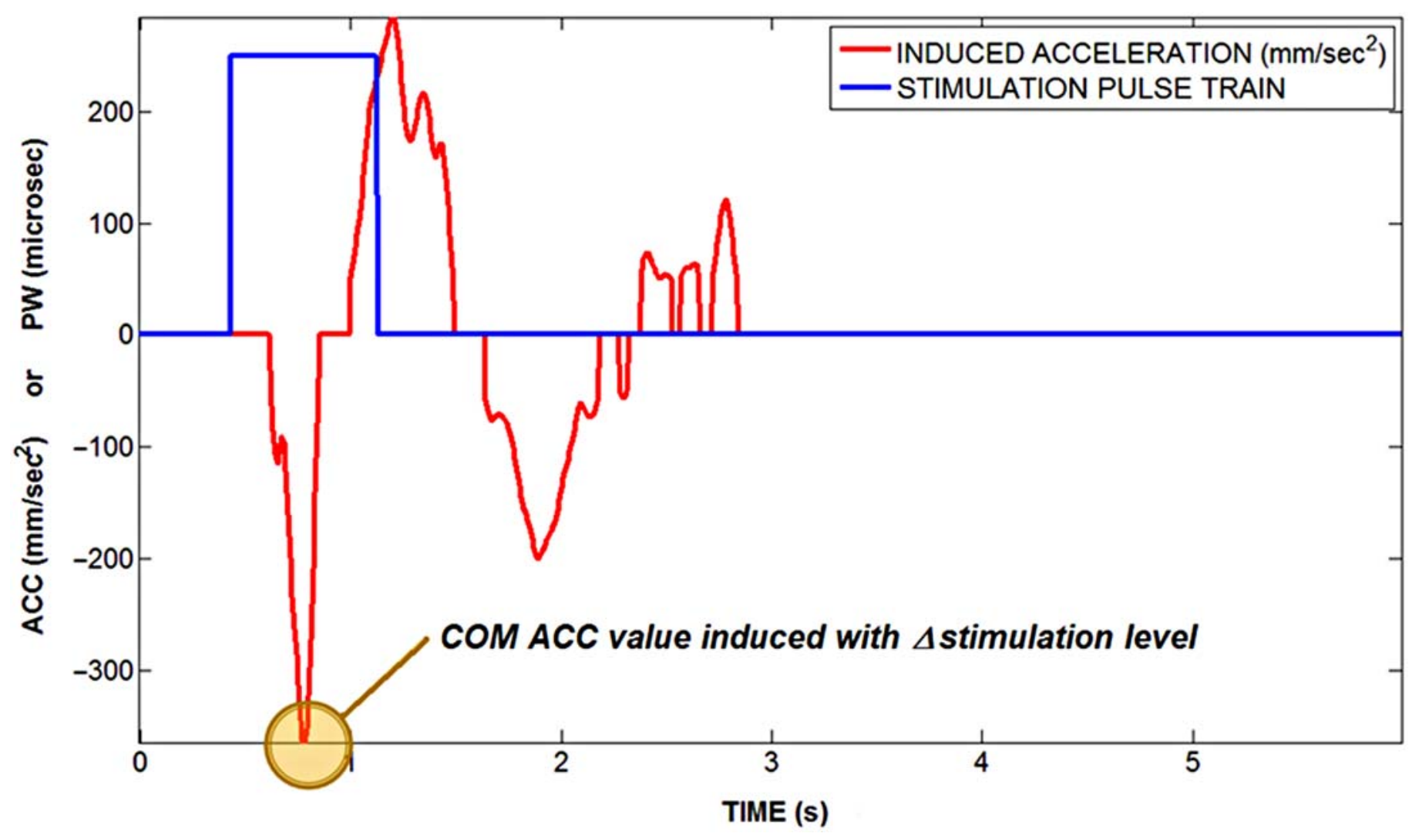

Figure 4.

Example of maximum total body center of mass (COM) acceleration (ACC) value induced in rightward $(+)$ direction in response to 750 $\mathrm{ms}$ pulse train of maximal pulse width (PW) of $250 \mu$ s stimulation to single muscle group (right gluteus medius). Absolute peak COM ACC observed during first $500 \mathrm{~ms}$ following onset of pulse train was recorded as induced value for trial. Note: Acceleration values $<50 \mathrm{~mm} / \mathrm{s}^{2}$ are set to zero. 
to maintain basic standing with FNS without acceleration feedback.

Given the initial states of the system corresponding to quiet erect standing, instantaneous changes in muscle activation forces would be proportional to the corresponding accelerations induced upon the system. Consequently, the instantaneous acceleration effects induced by individual muscles could be summed to yield the net system changes in acceleration, including net total body COM acceleration. Given these assumptions, the linear constraint equations to be satisfied by the optimizer to yield desired net $\mathrm{COM}$ acceleration $\left(A C C_{\mathrm{COM}}\right)$ targets were as follows:

$$
\begin{aligned}
& A C C_{\mathrm{COM}-\mathrm{AP}}=\sum_{i=1}^{N} a_{\mathrm{COM}-\mathrm{AP}, i} W_{i} \\
& A C C_{\mathrm{COM}-\mathrm{ML}}=\sum_{i=1}^{N} a_{\mathrm{COM}-\mathrm{ML}, i} W_{1}
\end{aligned}
$$

Changes in posture are assumed to be sufficiently small so as not to significantly alter the kinematic states of the system and shift the operation of individual muscles along their respective force-velocity and length-tension relationships [24]. This construction implies that individual changes in muscle activation, $W_{i}$, are proportional to the respective changes in stimulation, $M_{i}$. The validity of linearly mapping changes in individual stimulation levels to total body COM acceleration are investigated and discussed in Nataraj [20].

The COM acceleration was defined here by only two components, the AP and ML dimensions, with respect to a globally fixed anatomical reference frame. Each target COM acceleration component represented an optimization constraint that was equal to the weighted sum of the respective $a_{\mathrm{COM}}$ values in that dimension that can be induced by individual muscle groups ( $i$ ) from minimum activation levels. Each $W_{i}$ was the normalized (0 to 1 ) change in activation from minimum for each muscle group such that the weighted sum across all $(N=22)$ muscle groups must simultaneously satisfy both dimensional constraints. The third dimension of COM acceleration (in the inferior-superior direction) was assumed negligible provided the system abides by certain physical constraints observed for basic standing [25]. These constraints include the feet remaining grounded and sufficient extension moment to keep the knees from buckling. We have previously observed that these constraints were met in the presence of moderate perturbations (i.e., exter- nal force pulses $<300 \mathrm{~ms}$ duration and $<10 \%$ body weight amplitude) given sufficient minimal baseline stimulation to maintain erect standing.

Solution vectors for optimal muscle activations $(W)$ were determined as a constrained function minimum (MATLAB Optimization Toolbox, The MathWorks, Inc) that satisfies linear constraints of Equations (1) and (2) specifying the COM acceleration targets about quiet erect stance. The maximum target acceleration in each direction (i.e., fore/aft in the AP dimension, right/left for the ML dimension) were simply the sum of all the individual $a_{\mathrm{COM}}$ values recorded in that direction. These maximum target values were approximately $570,190,870$, and $490 \mathrm{~mm} / \mathrm{s}^{2}$ in the backward, forward, left, and right directions, respectively. A rectangular COM acceleration target space encompassing these limits about the zero-point for quiet standing (i.e., $A C C_{\mathrm{COM}}=0$ ) in increments of $20 \mathrm{~mm} / \mathrm{s}^{2}$ yielded a total of 2,584 targets. The solution vectors were optimized according to an objective function criterion that minimized the sum of squares of increases in stimulation pulse widths delivered (i.e., $\left.\Sigma\left(W_{i}\right)^{2}\right)$. Optimization parameters included a maximum of 10,000 iterations, constraint equation tolerance of $10 \mathrm{~mm} / \mathrm{s}^{2}$, and function tolerance of $1 \mu \mathrm{s}^{2}$. If the optimizer produced a solution that met the tolerance for both constraint equations for a given $A C C_{\mathrm{COM}}$, then that COM acceleration target solution was classified as "feasible." Only feasible solution points were retained for ANN training, testing, and validation.

\section{Step 3}

An ANN was trained to represent the synergy defined by the solution data from the optimization algorithm to map stimulation output as a function of acceleration input. The two components (AP, ML) of each feasible $A C C_{\mathrm{COM}}$ target served as the inputs. The corresponding solution vector $W$, presumed equal to the normalized optimal changes in stimulation $\left(M_{i}\right)$ from minimum levels for the 22 channels to produce $A C C_{\mathrm{COM}}$, served as the outputs. Each target set of 2 inputs and 22 outputs served as a single ANN data point. The data points were randomly assigned for ANN training (70\%), testing (20\%), and validation (10\%). The ANN was constructed with the Neural Network Toolbox in MATLAB. A three-layer (input, hidden, output), feedforward ANN structure was employed for its universal mapping capability of nonlinear functions [26]. The number of hidden layer neurons was determined to be 18 by heuristically finding the number of neurons providing the lowest mean 
squared error after 1,000 training epochs. All input and output data were normalized over $[-1,+1]$ before training using the mapminmax function with its default settings. The training function was the Levenberg-Marquardt algorithm [27]. A maximum of 10,000 epochs were specified for training in lieu of an early stopping criterion indicated as 250 consecutive epochs of increasing fitting error to the validation set. To enact optimal stimulation patterns for online use, the ANN outputs had to be de-normalized according to mapminmax settings, and then de-normalized from the $[0,1]$ range according to the respective $M_{\text {base }}$ and $M_{\max }$ for each muscle group in terms of stimulation pulse width (microseconds).

\section{Processing Acceleration Signals for Feedback Control}

Accelerometers were attached to the subject using double-sided tape. Preliminary experiments were performed to manually adjust the location of the sensors with the subject standing erect with continuous stimulation. Primary measurement axes of the sensors were aligned according to the presumed anatomical globally fixed reference frame. Sensors were calibrated at these positions to remove the effects of gravitational acceleration. It was assumed these sensors would be minimally misaligned during standing experiments. Pilot data showed that the subject underwent peak changes in orientation less than $10^{\circ}$ (relative to a globally fixed axis) at all segments except the reaching arm during stereotypical internally generated perturbations.

COM acceleration measurements below 15 percent of the maximum value on the controller map in each direction were set to zero before use as feedback inputs. This 15 percent threshold allowed the controller to only respond to larger disturbances. Trial and error observations and subject oral feedback indicated that the controller did not respond effectively to smaller disturbances and required notably more volitional effort to stabilize than constant stimulation. Application of this simple threshold essentially produced a low-pass filter effect without response delay to enact feedback control against larger amplitude disturbances.

\section{Tuning Center of Mass Acceleration Feedback Controller}

The controller map existed across two dimensions (AP and ML COM acceleration), which resulted in four distinct directions (front, back, right, left) having unique feedback gains. Each feedback gain for each direction across the acceleration-stimulation controller map required tuning for optimal standing performance. Initially tuning a directional gain required assigning a gain value, applying external perturbations to the subject in that direction using mounted linear actuators, and observing the resultant standing performance according to procedures described in Nataraj [20]. Improved standing performance was assessed as reducing the total UL loading applied by the subject to stabilize against the external perturbations. Total UL loading was calculated as the vector sum of the absolute net forces across all three dimensions applied by the subject at each side (i.e., left and right handles). The test gain values were bounded between 0 and 2.0 in each direction. The optimal gain values for resisting external perturbations were identified as those that resulted in the lowest average total UL loading in each respective direction. Best standing performance against external perturbations was initially designated as the optimal gain values.

Gains were readjusted for internal perturbations simply by multiplying the gains optimized for external perturbations by a corrective scaling factor in each dimension (AP, ML). The correction factor for each dimension was determined during internal perturbations generated by sliding the accelerometer across a smooth surface. Before the subject moved the accelerometer, each dimensional correction factor was applied to both directional gains in that dimension (e.g., AP correction factor multiplied both front and back directional gains), but all four gains used for resisting external perturbations were initially active. A total of 10 back and forth slide-shifts was performed in each dimension at the following correction factors: 0 , $0.25,0.50,0.75,1.00,1.50$, and 2.00 . Total UL loading for the support-side arm was continuously tracked over the course of each shift and hold until the next shift. The mean total UL loading across all 10 trial shifts was calculated for each correction factor value. A third-order polynomial fit was applied to the mean UL loading data as a function of correction factor value for each dimension. The minimum of the polynomial was taken to be the optimal correction factor to multiply both directional gains corresponding to that dimension. The net gain values following multiplication of the optimal correction factors were designated as optimal for internal perturbations.

\section{Testing Center of Mass Acceleration Feedback Controller}

Controller performance was determined in response to internal perturbations generated by moving the $1 \mathrm{~kg}$ jar 
in either the AP or ML dimensions with either the optimal gain feedback controller active or with maximal constant stimulation used clinically. All feedback gains were active and set equal to their respective optimal values during application of any of the test perturbations. The case of maximal constant stimulation corresponded to the pattern used by the subject for home operation. This constant stimulation pattern was classified as the "baseline" case against which controller operation was compared.

Two experimental sessions were performed for testing the feedback controller against internal perturbations. Across both sessions, 30 manual shifts were performed in each direction for both sliding and picking up/replacing the jar between the starting and target positions. Thus, a total of 60 trial shifts in each dimension was performed and repeated with the feedback controller active and with baseline constant stimulation.

\section{RESULTS}

\section{Acceleration-Stimulation Controller Map}

The maximum COM acceleration values (in both AP and ML dimensions) induced from quiet erect standing by a maximal increase in stimulation are shown for each muscle group listed in Table 1. Maximally increasing stimulation to muscle groups already being stimulated to provide basic standing support (i.e., minimum baseline levels $>0 \mu \mathrm{s}$ ) did not produce large acceleration changes. The mean value of the total COM acceleration induced was less than twice the standard deviation (SD) for all these muscle groups. Pilot attempts were made to produce greater changes in COM acceleration for these muscle groups by increasing stimulation frequency $(20$ to $30 \mathrm{~Hz}$ ) in addition to modulating pulse width. However, frequency modulation did not produce notably larger COM acceleration values. These muscle groups were strictly maintained at constant, clinically maximal levels for erect standing during all experiments and subsequently omitted in the construction of the controller acceleration-stimulation map, which only had 12 outputs corresponding to the remaining muscle groups.

The muscle groups that were retained for construction of the controller map and not relied on for basic standing support were activated by either implant (I) or surface (S) stimulation. Bilaterally, these muscle groups included tibialis anterior (I), triceps surae (I), gluteus medius (I), rectus femoris (S), thigh adductors (S), and erector spinae (S). Figure 5(a) shows the resultant feasible acceleration space for this particular subject following data optimization and ANN training with the maximum accelerationstimulation data for these 12 muscle groups. Also denoted are the lines connecting the zero-point to the largest stimulation-induced acceleration (i.e., 100\% response level) that can be generated by this participant in each of the four directions. Figure 5(b) depicts the normalized ANN output for stimulation levels of each of the 12 muscle groups targeted for feedback control across the feasible acceleration space. The ANN was highly capable of accurately mapping the input-output synergy represented by the optimized acceleration-stimulation data. The average normalized output error across all feasible targets for all muscle groups was only $0.0036 \pm 0.0698$.

The feasible acceleration space spanned an area of $6.08 \mathrm{e} 5 \mathrm{~mm}^{2} / \mathrm{s}^{4}$ with maximum directional values of $738 \mathrm{~mm} / \mathrm{s}^{2}$ in the left direction, $379 \mathrm{~mm} / \mathrm{s}^{2}$ in the right direction, $532 \mathrm{~mm} / \mathrm{s}^{2}$ in the backward direction, and $130 \mathrm{~mm} / \mathrm{s}^{2}$ in the forward direction. In the ML dimension, the ability to more greatly accelerate leftward than rightward is primarily due to this subject's relatively strong right-side gluteus medius, which produces hip abduction. This was confirmed by isometric dynamometer results for maximum joint moments produced using implanted stimulation [20]. The strong stimulated response of the right gluteus medius also elicited notable accelerations in the backward direction that could not be sufficiently balanced by stimulation of the remaining targeted muscles. As a result, the largest area of the feasible acceleration space was observed in the leftwardbackward quadrant.

In the AP dimension, the larger feasible acceleration space in the backward direction is a function of biomechanical constraints from erect standing and the musculature targeted for stimulation. From erect stance, accelerating the system COM forward can be accomplished by torso flexion with minimal posterior shifting of the lower body through activation of the abdominals, which are not currently targeted. Another way to accelerate the COM forward would be rapid hip and trunk extension that drives the pelvis and lower torso anteriorly. However, primary hip and trunk extensors targeted by implanted stimulation channels (i.e., semimembranosus, posterior adductor, gluteus maximus, erector spinae) were already recruited at near maximal levels for basic standing support. Tibialis anterior (I) and erector spinae (S), therefore, produced the greatest maximum COM acceleration values in the forward direction.

Modes to accelerate the COM backward from erect standing are not as compromised in this FNS system. The 
JRRD, Volume 49, Number 6, 2012

Table 1.

Maximum (peak) acceleration of total body center of mass (COM ACC) induced from quiet erect standing of subject with spinal cord injury, due to maximum increase in stimulation to individual muscle groups.

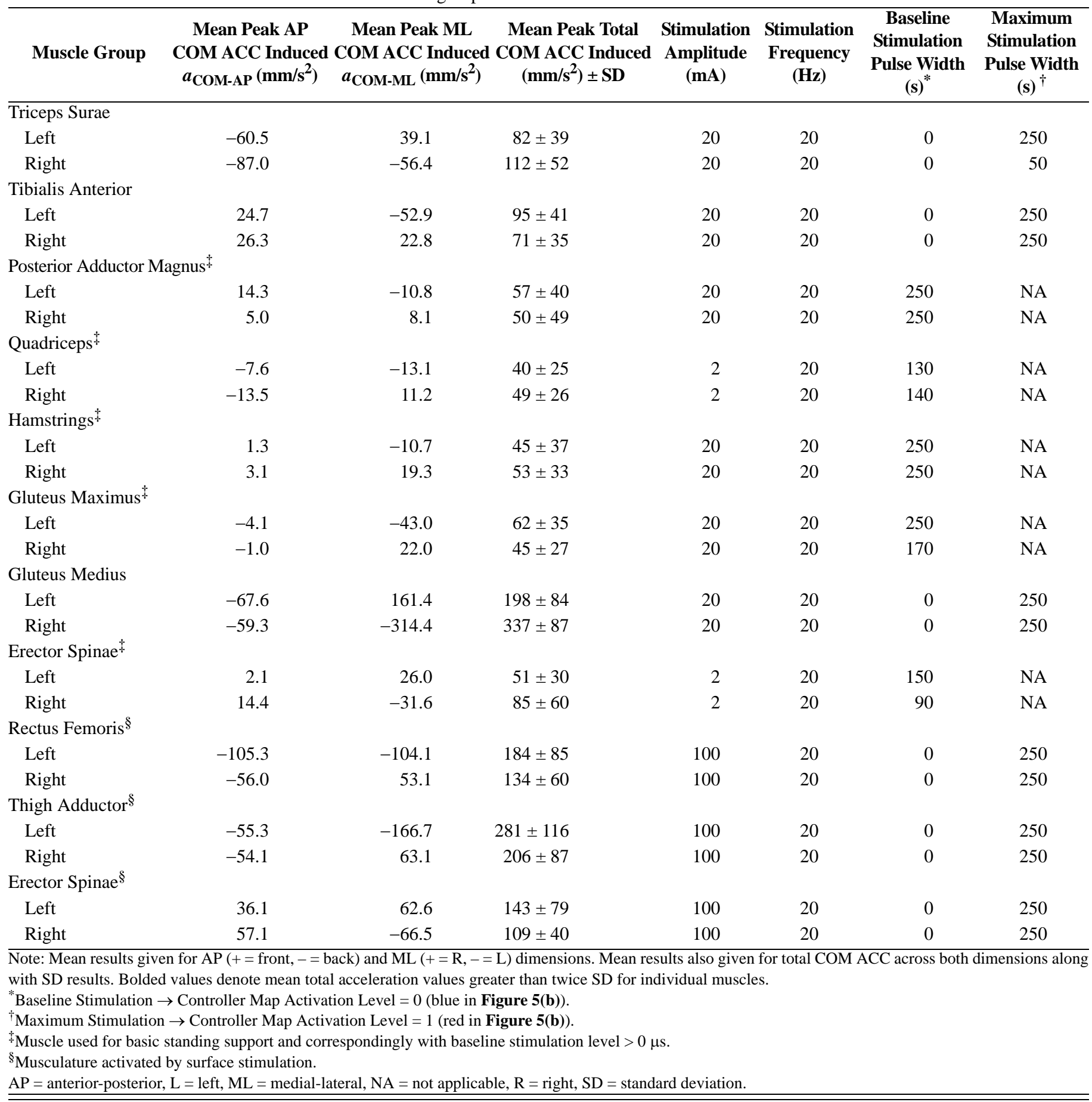

triceps surae (I) and rectus femoris (S) muscles were readily available to produce either ankle plantar flexion or rapid hip flexion to drive the COM posteriorly. Stimulation of gluteus medius (I) and thigh adductors (S) muscles also contributed to posterior acceleration. While these muscles primarily produce actions in the coronal plane with hip abduction/adduction, results from Table 1 demonstrate that they also induce notable maximum accelerations in the 

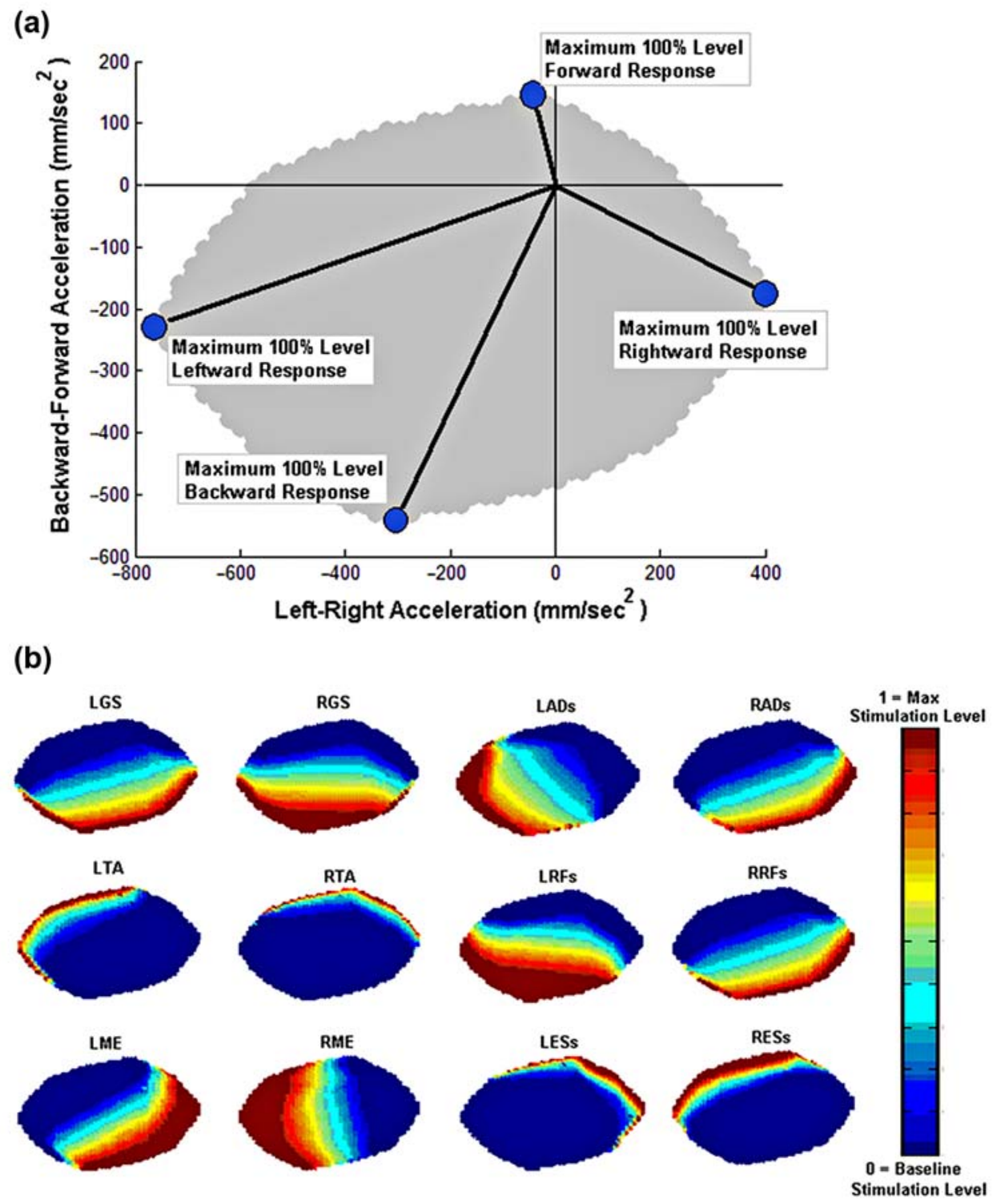

Figure 5.

(a) Feasible center of mass acceleration space for subject with spinal cord injury. (b) Corresponding optimal muscle stimulation levels for each of 12 muscle groups under functional neuromuscular stimulation feedback modulation. For muscle nomenclature, " $L$ " or "R" before muscle abbreviation denotes "left" or "right' and "s" after denotes "surface stimulation." AD = thigh adductors, ES = erector spinae, GS = triceps surae (soleus and gastrocnemius), Max = maximum, ME = gluteus medius, RF = rectus femoris, TA = tibialis anterior.

posterior direction. This was explained by the observation that their activation indirectly facilitated hip flexion on the contralateral side. Overall, the induced acceleration results in Table 1 and the ANN muscle activations maps in Figure 5 demonstrate the potency of hip muscles (gluteus medius, thigh adductors) to stabilize against medial-lateral disturbances and ankle muscles (gastrocnemius, tibialis anterior) to stabilize against anterior-posterior disturbances.
Given the maintenance of knees in extension for stable neuroprosthetic standing, these laboratory observations are consistent with ankle and hip strategies described for normative standing [17] and simulated neuroprosthetic standing [11-12]. In this experimental study, the directional sensitivity of applying more ankle versus hip strategies is emergent from the ANN since it has been trained on the data listed in Table 1. These data reflect the anatomically 
natural ability of hip abductors/adductors and ankle plantar flexors/dorsiflexors to generate larger accelerations in the ML and AP directions.

\section{Estimation of Center of Mass Acceleration for Feedback Control}

Table 2 lists the resultant linear regression model coefficients to estimate total body COM acceleration in the AP and ML dimensions during internal perturbations. In all cases, high correlation $\left(R^{2}>0.95\right)$ was observed between model output and the actual COM acceleration data as calculated from whole-body marker measurements. As expected, the largest positive regression coefficients were those multiplying inputs in the same direction as the component of COM acceleration dimension being estimated. The correlations of the fitted predictions for internal perturbations were higher than those reported for the external perturbations [20] along both dimensions, suggesting higher variability in the subject's response to unexpected external disturbances. For internal perturbations, adding outputs from a potential third sensor nearer the subject hand/object only marginally improved correlation. This suggests that motions of the arm during internal perturbations are largely coupled to those of the pelvis and torso. Sample regression outputs versus the actual COM acceleration during internal perturbations are shown in Figure 6.

\section{Tuning Feedback Controller Gains}

The tuning response data for readjusting the feedback gains against internal perturbations produced while the subject stabilized with one arm and volitionally slid an acceler- ometer with the other are shown in Figure 7. In the AP dimension, the optimal feedback gains for the front and back directions were adjusted (i.e., multiplied) by a correction factor of 0.6, which was the minimum of the respective third order polynomial fit. In the ML dimension, this correction factor was 1.6. The initial optimal feedback gains previously determined for resisting external perturbations as described in Nataraj [20] were 0.6, 0.7, 0.7, and 1.6 for the front, right, back, and left directions, respectively. Thus, the adjusted optimal feedback gains for internal perturbations in the front, right, back, and left directions were 0.36 , 1.12, 0.42 , and 1.76, respectively. The higher ML correction factor indicates that for unilateral stabilization in conjunction with contralateral volitional reaching, the subject was able to better employ stronger controller assistance in the ML dimension. This again could derive from the subject being more stable and stimulation effects having greater corrective capacity in the ML dimension. However, the observed reduction in total UL loading from constant stimulation (gain $=0$ ) at the optimal feedback gains was 26 percent lower in the ML dimension, versus 43 percent lower in the AP dimension. This result indicates the subject utilized the adjusted stimulation effects produced in the AP dimension more efficiently.

\section{Testing Feedback Controller Performance}

Figure 8 shows the mean total UL loading performance results with the optimally tuned feedback controller active versus baseline during sliding and picking up of the weighted jar in the AP and ML directions. These results are based on the average UL loading the subject applied

Table 2.

Linear regression model coefficients for estimation of center of mass acceleration (COM ACC) for sensor feedback during internal perturbations (Int Pert).

\begin{tabular}{|c|c|c|c|c|}
\hline \multirow{2}{*}{$\begin{array}{c}\text { Accelerometer Sensor } \\
\text { Feedback }\end{array}$} & \multicolumn{2}{|c|}{ AP COM ACC Int Pert } & \multicolumn{2}{|c|}{ ML COM ACC Int Pert } \\
\hline & 2 Sensors & 3 Sensors & 2 Sensors & 3 Sensors \\
\hline Torso-AP & 0.2128 & 0.2196 & -0.0177 & -0.0254 \\
\hline Torso-ML & 0.0524 & 0.0580 & 0.2743 & 0.2642 \\
\hline Torso-IS & -0.1022 & -0.1018 & -0.0388 & -0.0272 \\
\hline Pelvis-AP & 0.4654 & 0.5005 & -0.0035 & -0.0016 \\
\hline Pelvis-ML & -0.0275 & -0.0235 & 0.5248 & 0.5270 \\
\hline Hand-ML & NA & -0.0009 & NA & 0.0180 \\
\hline Hand-IS & NA & -0.0054 & NA & -0.0173 \\
\hline$R^{2}$ & 0.950 & 0.972 & 0.988 & 0.993 \\
\hline
\end{tabular}

$\mathrm{AP}=$ anterior-posterior, IS = inferior-superior, $\mathrm{ML}$ = medial-lateral, NA = not applicable. 
(a)

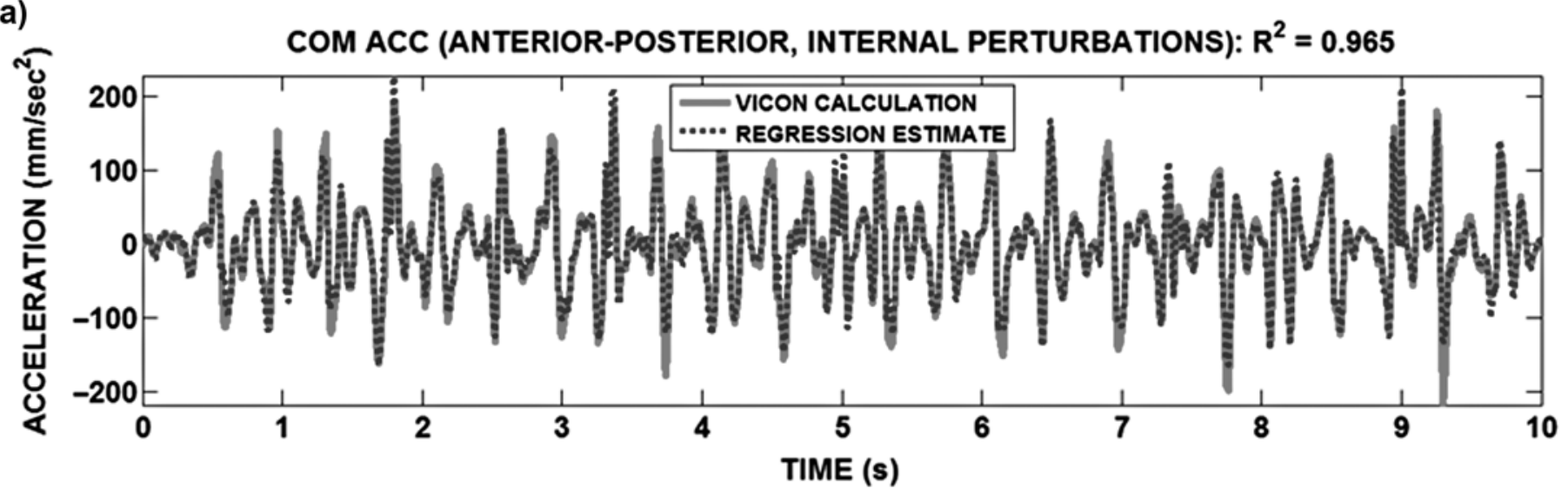

(b)

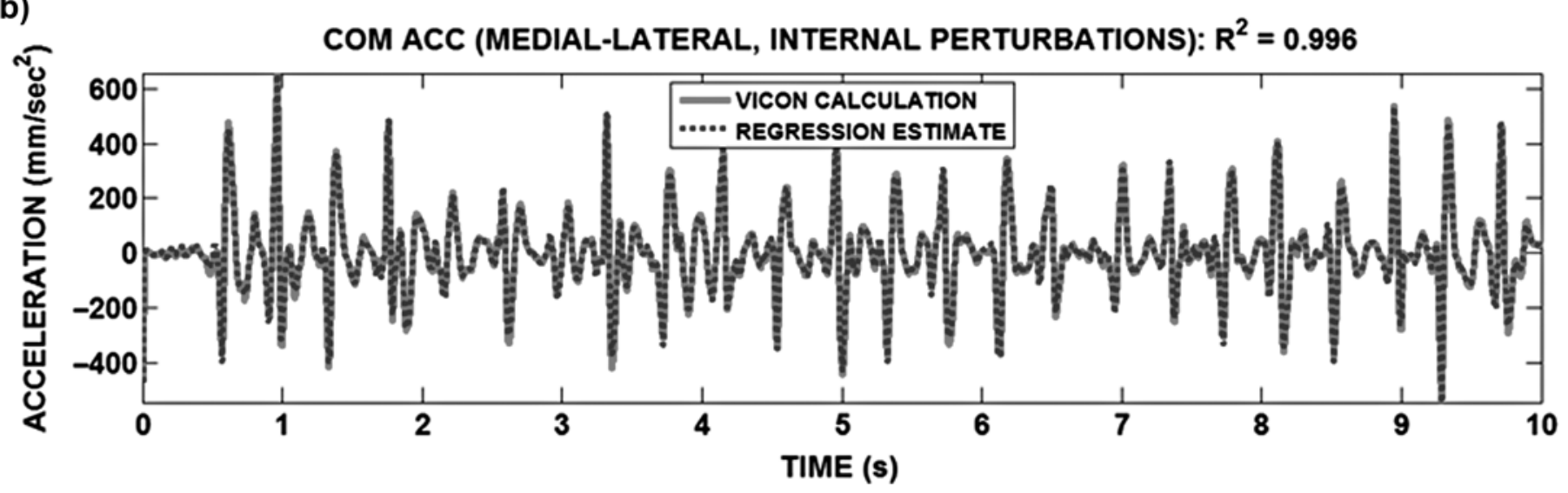

Figure 6.

Sample signals of total-body center of mass (COM) acceleration (ACC) estimated from linear regression model utilizing inputs from body-mounted (torso, pelvis) accelerometer measurements versus calculated from Vicon whole-body marker tracking. Samples shown for (a) anterior-posterior and (b) medial-lateral components of COM ACC.

across all test trials of each movement type. For each movement trial, the absolute changes in UL loading were tracked for a $1 \mathrm{~s}$ time interval after the velocity of the jar exceeded zero (i.e., initiation of jar movement corresponds to perturbation onset). Jar velocity was observed offline from differentiation of position data for a retroreflective marker placed on the jar. For tuning, total UL loading was continuously tracked across all shifts of each movement type. However, for these test results, discretization into individual trials was necessary for statistical comparisons of controller-active and baseline conditions.

Feedback control produced statistically significant reductions in UL loading compared with constant stimulation in both movement directions, regardless of whether sliding or lifting maneuvers were performed. As expected, UL loading was higher for lifting and replacing than slid- ing conditions. Picking up the jar along the ML dimension generated the greatest mean UL loading, which may be attributed to the extra effort required to move objects across midline. The mean change in UL loading required to stabilize while moving the jar object across all four movement cases ranged from 4.4 to $5.6 \mathrm{~N}$ with the feedback controller active and 6.2 to $8.4 \mathrm{~N}$ with baseline constant stimulation. The average reduction in UL loading with the controller active compared with baseline across all four cases was 27 percent.

The average SD was greater for the controller case than baseline ( 0.55 to $0.32 \mathrm{~N})$, indicating that the subject was responding more variably with modulation of stimulation. This differs from the case for external perturbations where active feedback control reduced the SD [20]. A direct comparison between these perturbation types is difficult 
(a) ANTERIOR-POSTERIOR

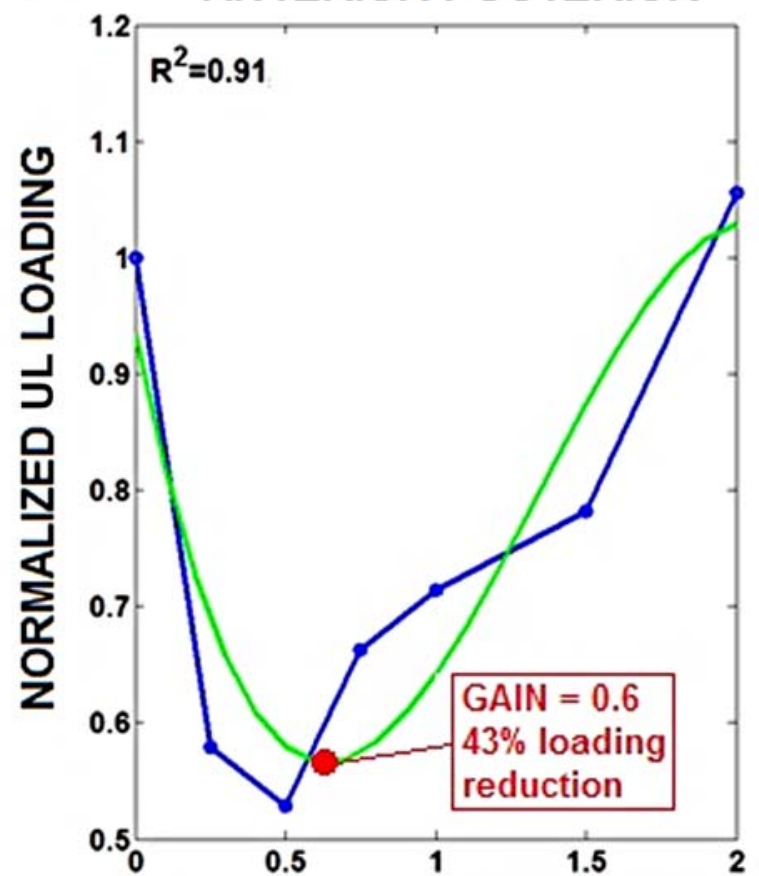

(b)
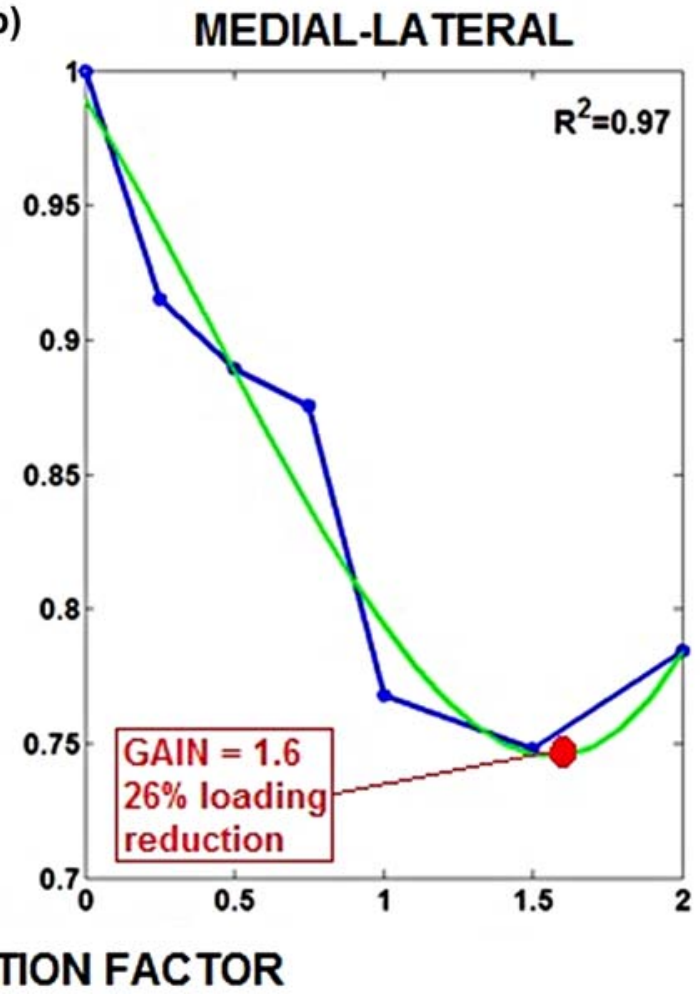

Figure 7.

Mean total upper-limb (UL) loading as function of gain correction factor during internal perturbations applied along either anteriorposterior or medial-lateral dimensions. Correction factor multiplied both directional feedback gain values in that dimension originally determined as optimal for external perturbations [17]. Third-order polynomial fit (i.e., green trace) was applied to each dimensional data set, and polynomial minimum was identified as corresponding "optimal" gain correction factor.

since the changes in UL loadings generated during internal perturbations are much smaller than those for external perturbations (see Nataraj [20] for sample results). However, the subject may have more difficulty accommodating to variations in stimulation when undergoing a more active task. In resisting external perturbations, the subject reactively stabilized against unknown disturbances. For internal perturbations, the subject simultaneously stabilized and volitionally generated functional movements. The subject may be able to further accommodate to stimulation effects during internal perturbations with practice that would consequently reduce the observed SD.

The average increases in stimulation levels delivered in response to internal perturbations are shown in Figure 9(a). The average was taken over the $1 \mathrm{~s}$ interval immediately following perturbation onset in all cases. The average normalized stimulation level (i.e., average ANN output) was less than 0.4 for every muscle group across every perturbation condition, indicating that the controller took finite actions in response to the perturbations. Multiplying the nor- malized increase in stimulation by the maximum total COM acceleration value that can be induced for a muscle yields the contribution to the controller response by that muscle. The relative contribution of a muscle is this value divided by the sum of all contributions from all muscles. The relative contributions for all the stimulated muscle groups during internal perturbations are shown in Figure 9(b).

The triceps surae, tibialis anterior, erector spinae, and rectus femoris muscles had higher contributions to controller responses for disturbances in the AP dimension. The gluteus medius and thigh adductor muscles had higher contributions to controller response for disturbances in the ML dimension. These results are anatomically consistent. The triceps surae and tibialis anterior mediate ankle plantar flexion/dorsiflexion and rectus femoris and erector spinae produce notable flexion and extension changes at the pelvis and lower torso. These actions typically produce movements primarily along the sagittal plane. Gluteus medius and thigh adductor muscles mainly generate hip 


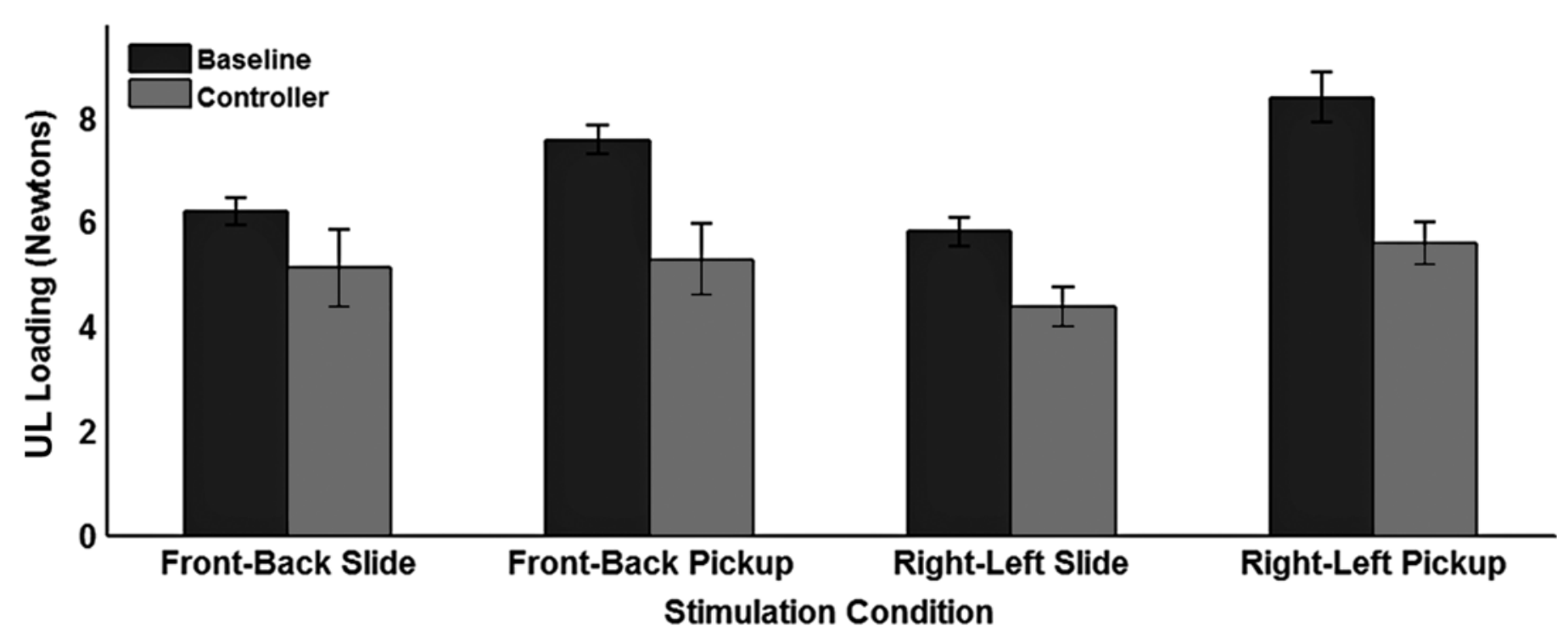

Figure 8.

Mean total upper-limb (UL) loading to stabilize with one arm against internal perturbations generated while volitionally moving $1 \mathrm{~kg}$ jar with other arm. Jars were moved by either sliding or picking up/placing down in front-back (anterior-posterior dimension) or rightleft (medial-lateral dimension) paired directions.

abduction/adduction moments to produce shifts from erect standing primarily along the coronal plane.

The right and left gluteus medius muscles alone contributed on average to greater than 40 percent of the controller response for internal perturbations. The other muscle group that was generally very active across all perturbations was surface-stimulated erector spinae. Right and left surface-stimulated erector spinae contributed to greater than 20 percent of the controller response for either external or internal perturbations. These relatively high contributions are direct consequences of these muscle groups being capable of inducing strong changes in COM acceleration following stimulation. These trends for muscle contributions are similar to those observed for external perturbations [18], which suggest that the optimized muscle actions are more a function of muscle force generating capacity than the mode of balance perturbation. Sample controller operation results, such as changes in COM acceleration, COM position, UL loading, and muscle stimulation levels for individual external and internal perturbation trials, can be found in Nataraj [20].

\section{DISCUSSION}

This study investigated total body COM acceleration as feedback for comprehensive 3-D control of standing by
FNS with an SCI subject in the laboratory. It advances the scope of clinical feasibility compared with previous investigations that explored FNS standing control across single planes of movement [28-29]. While they demonstrated modest potential for unsupported standing, prior studies applied bracing constraints that are clinically unviable. Further developments of FNS standing control systems should implement features that facilitate clinical acceptance while providing effective clinical performance simultaneously in all three dimensions.

While 22 muscle groups were targeted for stimulation in this system, only 12 muscle groups were under feedback control for balance and the remaining 10 were under constant stimulation and provided basic standing support. This functional distribution was necessary because of difficulty in producing dynamic changes in standing posture by increasing stimulation to muscles already recruited for basic support. This could be due to inability to activate additional muscle fibers or that these particular muscle groups may be at disadvantageous positions with respect to their force-length properties [24]. While this subject assumed a desirably erect posture during quiet standing, extensor muscles about the knees, hips, and trunk may be too short to produce the additional forces required. These included the quadriceps, hamstrings, gluteus maximus, posterior adductor magnus, and erector spinae bilaterally, 
(a)

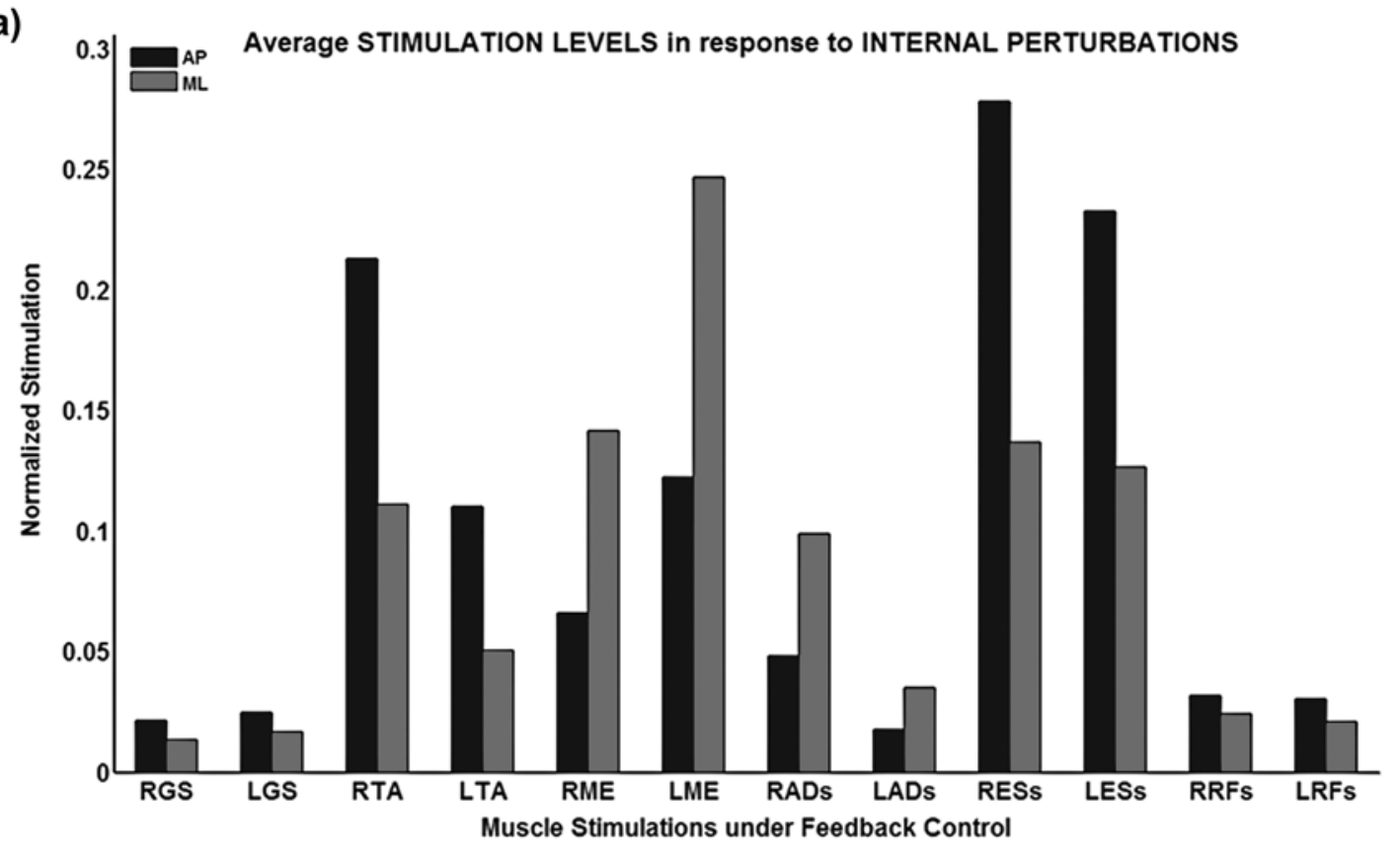

(b)

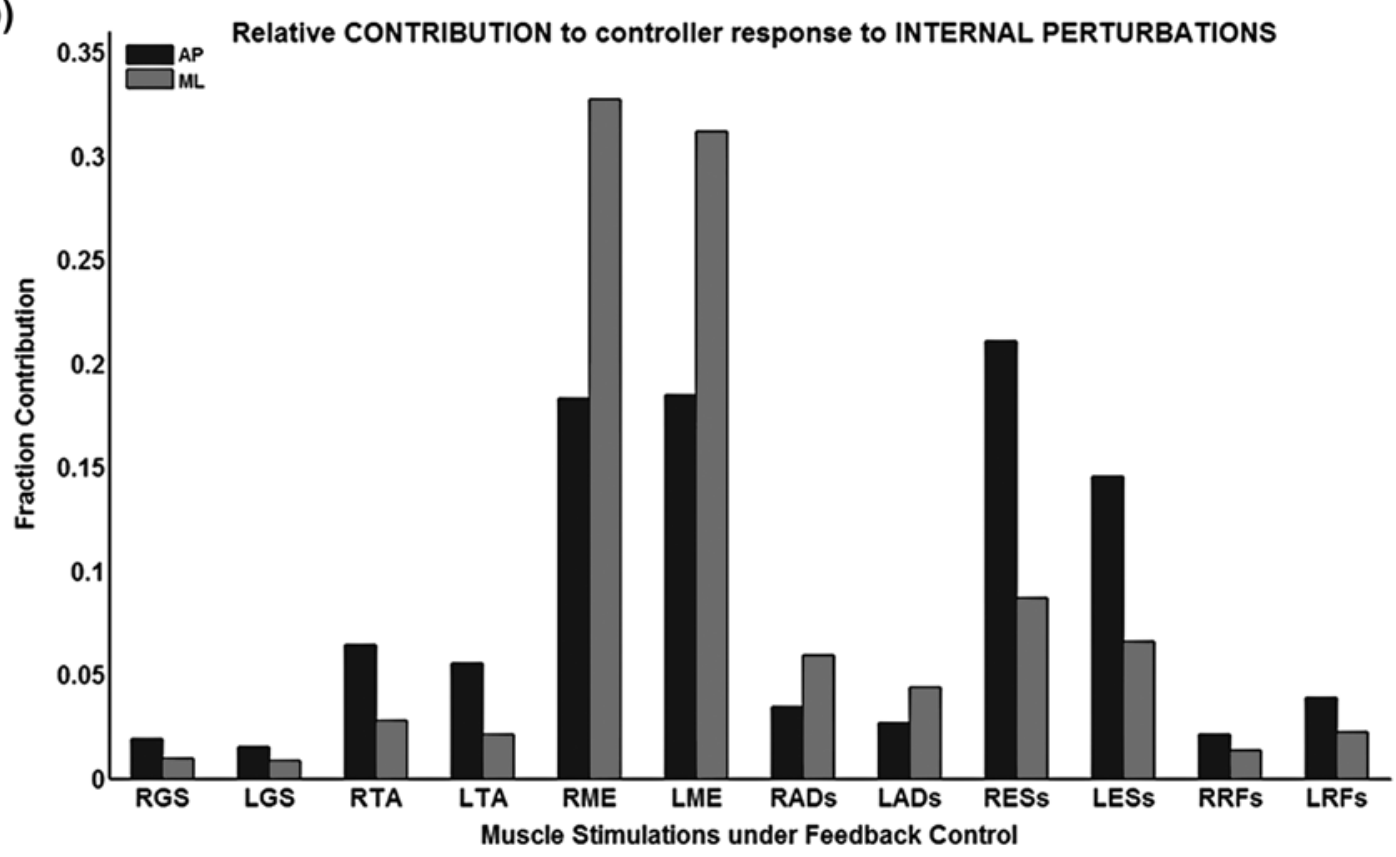

Figure 9.

(a) Average normalized stimulation levels (i.e., artificial neural network outputs) during controller response to internal perturbations in each dimension, anterior-posterior (AP) and medial-lateral (ML). Average was taken over $1 \mathrm{~s}$ interval following perturbation onset. (b) Fraction contribution to total COM acceleration response being induced by controller via stimulation of individual muscle groups. For muscle nomenclature, "L" or "R" before muscle abbreviation denotes "left" or "right" and "s" after denotes "surface stimulation." $\mathrm{AD}=$ thigh adductors, $\mathrm{ES}=$ erector spinae, $\mathrm{GS}=$ triceps surae (soleus and gastrocnemius), $\mathrm{ME}=$ gluteus medius, $\mathrm{RF}=$ rectus femoris, $\mathrm{TA}=$ tibialis anterior. 
which were all activated by the implanted stimulator. However, despite only 12 muscle groups for feedback control of balance, an improvement in standing performance could be assessed. Furthermore, the knees were desirably maintained in extension not only because of clinical objectives of safety to ensure stable clinical standing, but also because of biomechanical constraints for balance control against perturbations. This has been previously reported for both normative [15] and simulated neuroprosthetic standing [11-12,20].

The results of this study demonstrated that COM acceleration feedback is a clinically viable alternative to joint feedback for control of FNS standing during volitionally generated postural perturbations. Firstly, results from the linear regression model produced highly accurate estimates of total body COM acceleration for feedback during internally perturbed standing using only two body-mounted accelerometers, one at the pelvis and one at the torso. Changes in orientation at the pelvis and torso were minimal relative to the globally fixed anatomical reference frame such that significant aliasing errors were not observed. Since analog accelerometers record accelerations on a local reference frame, notable changes in sensor orientation would cause acceleration components, including gravity, to be erroneously recorded along incorrect measurement axes. However, the high degree of estimation accuracy with a simple linear regression model using inputs from only two accelerometers suggests this is not the case in this application. Because the reaching arm undergoes significant changes in acceleration relative to the other body segments during standing, two separate linear regression models were determined for internal disturbances. The second included inputs from not only the pelvis and torso accelerometers, but also from an additional accelerometer placed near the hand moving the object. However, the improvement in estimation accuracy using a third accelerometer was negligible. Thus, the first linear regression model using inputs from only the pelvis and torso appears to sufficiently estimate COM acceleration for internal disturbances. This is an important conclusion for practical usage of this acceleration-based control system. This study explored initial feasibility by ensuring that the "hand" accelerometer maintained its orientation, which prevented feedback aliasing from this sensor. However, for home use, it is highly likely that a sensor placed near the reaching hand would undergo significant changes in orientation while reaching and manipulating objects.
Secondly, the dynamic responsiveness of COM acceleration feedback was preserved, making it inherently superior to joint position-based feedback in compensating for delays in muscle force generation following stimulation onset [30] and capturing rapid perturbation effects without large changes in postural configuration. The sensor signals did not require significant processing (e.g., low-pass filtering) before controller input because the $20 \mathrm{~Hz}$ sampling rate was sufficiently high for this application. Accelerometer measurement values were simply cut off based on a magnitude threshold so controller action was initiated only for larger postural changes. Advanced signal processing techniques could be investigated in the future to determine additional performance benefit, but the sensor signals were readily utilized for high responsiveness without notable group delays with this relatively simple processing scheme.

Finally, an optimally tuned COM acceleration feedback controller produced notably improved standing performance against postural perturbations compared with clinical levels of constant stimulation. Feedback control reduced UL loading by 27 percent against internal perturbations. While external perturbations provide a systematic and repeatable way to initially test and develop a standing balance system, they may not be as relevant clinically as internally generated perturbations from functional voluntary movements. In this study, internal perturbations were restricted to systematic shifting of an object across a level surface strictly in either the AP or ML dimensions. In the future, functional reaching activities that include manipulating objects of varying weight and shape in more complex 3-D motions should be examined [31]. However, the internal perturbation tests presented in this study represent important initial steps in demonstrating feasibility and further assessing controller performance.

Furthermore, it may be possible to produce significantly greater reductions in UL loading with larger muscle forces that are still achievable with current clinical paradigms for stimulation and muscle conditioning. In simulation [20], 40 to 70 percent reductions in UL loading were observed for a model that employed moderately greater joint moments than this subject. These joint moments were reported to be typical when maximally stimulating paralyzed musculature using a combination of surface and percutaneous electrodes and measuring while user is seated on a Biodex ${ }^{\circledR}$ (Biomedical Systems, Inc; Shirley, New York) dynamometer [32]. However, implantable stimulators produce clinically reliable responses and improved cosmesis for the users [21]. This system provides a test-bed that 
includes multiple muscle groups to provide both static and dynamic function. In the future, additional subjects should be recruited to provide a more thorough evaluation of this clinical system beyond the case study reported here.

While this study demonstrates the feasibility of this approach in a laboratory environment, future clinical development would rely on further streamlining of these methods. The number of sessions undergone for this pilot study is considerably more than will be required for typical clinical deployment. Once methods are finalized for efficiency, a single session would be required for collection of training data and another for initial controller tuning and testing. Retuning in a well-controlled laboratory environment would be periodically performed as is typical and reasonable for these clinical systems that employ minimal instrumentation.

The concept of muscle-induced accelerations has been used to assess actions of individual muscles in analyzing motion $[10,25]$ and for constructing FNS control systems in simulation [12]. In creating the experimental formulation for FNS feedback control of standing in this study, optimization constraints were specified to linearly relate changes in stimulation levels for targeted muscle groups according to corresponding changes in COM acceleration observed from quiet, erect stance. The resultant optimization data were used to successfully train an ANN to represent an input-output synergy for feedback control of FNS standing balance. The original formulation assumed that instantaneous changes in COM acceleration resulted from instantaneous changes in muscle activation levels, but the experimental construction relied on utilizing maximum acceleration values observed within a window of time following initial stimulation onset. This introduces potential compromises in map accuracy and consistency because of changes in postural configuration occurring in the presence of excitation-activation dynamics before observation of the maximum COM acceleration value. These effects were negligible given the minimal postural changes that occurred over the short time intervals of the perturbations. Furthermore, it was demonstrated that the resultant map could be effectively tuned to yield improved standing performance against internal perturbations.

Minimizing instrumentation and tuning this system in the laboratory were practical because standing synergies under perturbation could be simplified $[17,18,20,33]$ and this feedback control system only operated along two dimensions (AP, ML). Enacting control in the inferiorsuperior dimension was not necessary given its coupling to the other two dimensions assuming basic standing constraints [25]. These constraints would be violated in cases of system collapse such as knee buckling, which has been previously explored for FNS standing with accelerationbased detection of knee unlock [34]. However, this consideration was beyond the scope of this study, which primarily aimed to create a control system to enact comprehensive balance about basic standing. In any case, the observed simplification in standing synergies is the basis from which it was possible to accurately capture changes in COM acceleration utilizing accelerometer inputs from only two body-mounted accelerometers. Ultimately, we demonstrated that a linear regression model could produce a sensor-based estimate of COM acceleration. Model output closely matched the more rigorous measurement based on kinematic tracking of all major body segments using Vicon motion capture. Whether two or three sensors were employed, the regression output matched the presumed gold standard with a correlation coefficient $>0.95$ in both the AP and ML dimensions. This estimation accuracy may degrade with more complex arm movements (e.g., higher lifting, motions coupled along AP and ML) and lifting of heavier objects that would shift effective COM toward the motions of the arms. However, given simplified standing synergies that notably couple motions of the remaining body segments (e.g., head, torso, pelvis, lower limbs) that largely compose total body COM, arm effects are likely to be consistently mitigated. Future studies could confirm this notion by applying the presented techniques to additional subjects with varying body types who perform a variety of functional standing and reaching tasks [31].

Optimal gains for this control system were initially determined for external perturbations by using a laboratory perturbation system employing linear actuators described in Nataraj [20]. The perturbation system was capable of applying discrete force-pulse disturbances that instantly induced accelerations on the subject COM in specific directions. These procedures enabled focal tuning of feedback gains in each direction. In this study, we simply adjusted those gains according to a scaling factor to both directions in each dimension. This was necessary because moving an object along one dimension produced significant accelerations in both directions of that dimension for both initially moving the object and then bringing it to rest, regardless of the initial direction of motion.

While the procedures to tune the gains are appropriate for initial laboratory evaluations, they may be inconvenient for periodic retuning during long-term home use. 
Future work should focus on developing simpler and more robust methods to determine the optimal feedback gains. Previous studies have outlined the importance of adapting system parameters according to time-varying muscle output [35]. Adaptive techniques would be critical to ensure long-term success and clinical acceptance of these neuroprosthetic systems by minimizing the cycle time to develop and test in the laboratory before home deployment. Thus, it would be valuable to demonstrate the feasibility for a method that potentially retunes a standing control system with minimal effort. This would facilitate long-term optimal performance and possibly provide a gateway for addressing the issue of muscle fatigue with FNS [36].

While a position-based system such as joint control is theoretically necessary for "hands-free" standing, technological advances in stimulation of paralyzed musculature have not yet produced such a solution clinically despite notable previous work in joint-based control [4-8]. This study approach aims to reduce the reliance on volitional UL loading to stabilize the position and velocity states of the system against and in providing a solution that is clinically relevant. It does not constrain motion to single planes, does not require bracing to assume a simpler version of the standing system, and facilitates functional 3-D standing. Furthermore, the basis of clinical viability for this type of system stems from usage of only two small, body-mounted sensors that need to be added to a current neuroprosthetic system [21] for standing that is already being used. Ultimately, it maximizes the dynamic capabilities of residual stimulated musculature not relied on for stable standing as assessed by clinical observation. While controller stability has not been established for this "soft computing" controller structure, performance has been demonstrated under laboratory observation. Further development of practical, clinically relevant neuroprosthetic systems such as the one presented here should continue to focus on methods that optimize standing performance by demonstrating additional reductions in UL loading. Ideally, these methods would converge with proposed jointfeedback systems that require constraints but produce demonstrable theory for a stable, hands-free solution and means to focally adapt against muscle fatigue [9].

\section{CONCLUSIONS}

This study investigated COM acceleration feedback to produce a clinically viable solution for closed-loop control of FNS standing and maintenance of postural balance against internal perturbations that are volitionally generated during reaching actions. This feedback control system was created using optimization to linearly relate changes in muscle stimulation to corresponding changes in acceleration of the total body COM in the AP and ML dimensions. Only two body-mounted accelerometers were required to accurately estimate COM acceleration for feedback control. Against internal perturbations, feedback control notably reduced the UL loading required to stabilize compared with clinically determined constant stimulation levels. Future developments should focus on methods that minimize procedures to retune feedback gains and that further utilize reduction of UL loading as a performance metric.

\section{ACKNOWLEDGMENTS}

\author{
Author Contributions: \\ Intellectual conceptualization and design: R. Nataraj. \\ Intellectual support and guidance: M. L. Audu, R. J. Triolo. \\ Data collection: R. Nataraj, M. L. Audu. \\ Data analysis: R. Nataraj. \\ Document writing: R. Nataraj. \\ Document editing: M. L. Audu, R. J. Triolo. \\ Financial support: R. J. Triolo.
}

Financial Disclosures: The authors have declared that no competing interests exist.

Funding Sources: This material was based on work supported by the National Institutes of Health (grant R01 NS040547-04A2).

Additional Contributions: This work was conducted at the Motion Study Laboratory at the Louis Stokes Cleveland VAMC in conjunction with Case Western Reserve University. Research at the VAMC strives to improve quality of life of veterans with disabilities. Raviraj Nataraj is now a postdoctoral research fellow at the Cleveland Clinic Lerner Research Institute.

Institutional Review: The subject signed informed consent forms approved by the institutional review board of the Louis Stokes Cleveland VAMC.

Participant Follow-Up: The authors do not plan to inform the participant of the publication of this study. However, the participant has been encouraged to check the study Web site for updated publications.

\section{REFERENCES}

1. Triolo RJ, Bogie K. Lower extremity applications of functional neuromuscular stimulation after spinal cord injury. 
Top Spinal Cord Inj Rehabil. 1999;5(1):44-65. http://dx.doi.org/10.1310/UXUE-5L1R-WKKV-1RGP

2. Kralj A, Bajd T, Turk R. Electrical stimulation providing functional use of paraplegic patient muscles. Med Prog Technol. 1980;7(1):3-9. [PMID:6966735]

3. Kralj A, Bajd T. Functional electrical stimulation: standing and walking after spinal cord injury. Boca Raton (FL): CRC Press; 1989.

4. Jaeger RJ. Design and simulation of closed-loop electrical stimulation orthoses for restoration of quiet standing in paraplegia. J Biomech. 1986;19(10):825-35.

[PMID:3782165]

http://dx.doi.org/10.1016/0021-9290(86)90133-8

5. Moynahan M, Chizeck HJ. Characterization of paraplegic disturbance response during FNS standing. IEEE Trans Rehabil Eng. 1993;1(1):43-48. http://dx.doi.org/10.1109/86.242407

6. Chizek HJ, Kobetic R, Marsolais EB, Abbas JJ, Donner IH, Simon E. Control of functional neuromuscular stimulation systems for standing and locomotion in paraplegics. Proc IEEE. 1988;76(9):1155-65. http://dx.doi.org/10.1109/5.9661

7. Abbas JJ, Chizeck HJ. Feedback control of coronal plane hip angle in paraplegic subjects using functional neuromuscular stimulation. IEEE Trans Biomed Eng. 1991;38(7): 687-98. [PMID:1879862]

http://dx.doi.org/10.1109/10.83570

8. Hunt KJ, Gollee H, Jaime RP. Control of paraplegic ankle joint stiffness using FES while standing. Med Eng Phys. 2001;23(8):541-55. [PMID:11719077] http://dx.doi.org/10.1016/S1350-4533(01)00089-3

9. Kobravi H-R, Erfanian A. A decentralized adaptive fuzzy robust strategy for control of upright standing posture in paraplegia using functional electrical stimulation. Med Eng Phys. 2012;34(1):28-37. [PMID:21764350] http://dx.doi.org/10.1016/j.medengphy.2011.06.013

10. Zajac FE, Gordon ME. Determining muscle's force and action in multi-articular movement. Exerc Sport Sci Rev. 1989;17:187-230. [PMID:2676547]

11. Nataraj R, Audu ML, Kirsch RF, Triolo RJ. Comprehensive joint feedback control for standing by functional neuromuscular stimulation-a simulation study. IEEE Trans Neural Syst Rehabil Eng. 2010;18(6):646-57. [PMID:20923741] http://dx.doi.org/10.1109/TNSRE.2010.2083693

12. Nataraj R, Audu ML, Kirsch RF, Triolo RJ. Center of mass acceleration feedback control for standing by functional neuromuscular stimulation: a simulation study. J Rehabil Res Dev. 2012;49(2):279-96. http://dx.doi.org/10.1682/JRRD.2010.12.0235

13. Mayagoitia RE, Lötters JC, Veltink PH, Hermens H. Standing balance evaluation using a triaxial accelerometer. Gait Posture. 2002;16(1):55-59. [PMID:12127187] http://dx.doi.org/10.1016/S0966-6362(01)00199-0
14. Moe-Nilssen R, Helbostad JL. Trunk accelerometry as a measure of balance control during quiet standing. Gait Posture. 2002;16(1):60-68. [PMID:12127188] http://dx.doi.org/10.1016/S0966-6362(01)00200-4

15. Betker AL, Moussavi ZM, Szturm T. Center of mass approximation and prediction as a function of body acceleration. IEEE Trans Biomed Eng. 2006;53(4):686-93. [PMID:16602575] http://dx.doi.org/10.1109/TBME.2006.870222

16. Pai YC, Patton J. Center of mass velocity-position predictions for balance control. J Biomech. 1997;30(4):347-54. [PMID:9075002] http://dx.doi.org/10.1016/S0021-9290(96)00165-0

17. Horak FB, Nashner LM. Central programming of postural movements: adaptation to altered support-surface configurations. J Neurophysiol. 1986;55(6):1369-81. [PMID:3734861]

18. Krishnamoorthy V, Goodman S, Zatsiorsky V, Latash ML. Muscle synergies during shifts of the center of pressure by standing persons: identification of muscle modes. Biol Cybern. 2003;89(2):152-61. [PMID:12905043] http://dx.doi.org/10.1007/s00422-003-0419-5

19. Winter DA. Biomechanics and motor control of human movement, 2nd ed. Toronto (Canada): John Wiley \& Sons; 1990.

20. Nataraj R. Feedback control of standing balance by functional neuromuscular stimulation following spinal cord injury [dissertation]. [Cleveland]: Case Western Reserve University; 2011. $467 \mathrm{p}$.

21. Bhadra N, Kilgore KL, Peckham PH. Implanted stimulators for restoration of function in spinal cord injury. Med Eng Phys. 2001;23(1):19-28. [PMID:11344004] http://dx.doi.org/10.1016/S1350-4533(01)00012-1

22. Kaiser JF, Reed WA. Data smoothing using low-pass digital filters. Rev Sci Instrum. 1977;48(11):1447-55.

http://dx.doi.org/10.1063/1.1134918

23. Winter DA. ABC (anatomy, biomechanics, control) of balance during standing and walking. Waterloo (Canada): Waterloo Biomechanics; 1995.

24. Zajac FE. Muscle and tendon: properties, models, scaling, and application to biomechanics and motor control. Crit Rev Biomed Eng. 1989;17(4):359-411. [PMID:2676342]

25. Kuo AD, Zajac FE. A biomechanical analysis of muscle strength as a limiting factor in standing posture. J Biomech. 1993;26(Suppl 1):137-50. [PMID:8505348] http://dx.doi.org/10.1016/0021-9290(93)90085-S

26. Haykin SS. Neural networks: a comprehensive foundation, 2nd ed. Upper Saddle River (New Jersey): Prentice Hall; 1999.

27. Hagan MT, Menhaj MB. Training feedforward networks with the Marquardt algorithm. IEEE Trans Neural Netw. 
1994;5(6):989-93. [PMID:18267874]

http://dx.doi.org/10.1109/72.329697

28. Matjaci Z, Bajd T. Arm-free paraplegic standing-part II: experimental results. IEEE Trans Rehabil Eng. 1998;6(2): 139-50. [PMID:9631321] http://dx.doi.org/10.1109/86.681179

29. Gollee H, Hunt KJ, Wood DE. New results in feedback control of unsupported standing in paraplegia. IEEE Trans Neural Syst Rehabil Eng. 2004;12(1):73-80.

[PMID:15068190] http://dx.doi.org/10.1109/TNSRE.2003.822765

30. Schutte LM, Rodgers MM, Zajac FE, Glaser RM. Improving the efficacy of electrical stimulation-induced leg cycle ergometry: an analysis based on a dynamic musculoskeletal model. IEEE Trans Rehabil Eng. 1993;1(2):109-25. http://dx.doi.org/10.1109/86.242425

31. Triolo RJ, Reilley BW, Freedman W, Betz RR. Development and standardization of a clinical evaluation of standing function. IEEE Trans Rehabil Eng. 1993;1(1):18-25. http://dx.doi.org/10.1109/86.242404

32. Kobetic R, Marsolais EB. Synthesis of paraplegic gait with multichannel functional neuromuscular stimulation. IEEE Trans Biomed Eng. 1994;2(2):66-79.

33. Matjacic Z. Control of ankle and hip joint stiffness for armfree standing in paraplegia. Neuromodulation. 2001;4(1): 37-46. [PMID:22151571] http://dx.doi.org/10.1046/j.1525-1403.2001.00037.x
34. Veltink PH, Franken HM. Detection of knee unlock during stance by accelerometry. IEEE Trans Rehabil Eng. 1996; 4(4):395-402. [PMID:8973965] http://dx.doi.org/10.1109/86.547941

35. Abbas JJ, Gillette JC. Using electrical stimulation to control standing posture. IEEE Contr Syst Mag. 2001;21(4): 80-90. http://dx.doi.org/10.1109/37.939946

36. Matjai Z, Hunt K, Gollee H, Sinkjaer T. Control of posture with FES systems. Med Eng Phys. 2003;25(1):51-62. [PMID:12485786] http://dx.doi.org/10.1016/S1350-4533(02)00115-7

Submitted for publication July 17, 2011. Accepted in revised form November 2, 2011.

This article and any supplementary material should be cited as follows:

Nataraj R, Audu ML, Triolo RJ. Center of mass acceleration feedback control of functional neuromuscular stimulation for standing in presence of internal postural perturbations. J Rehabil Res Dev. 2012;49(6):889-912.

http://dx.doi.org/10.1682/JRRD.2011.07.0127

ResearcherID: Raviraj Nataraj: G-4535-2010

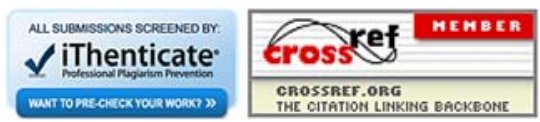


